\title{
A STABLE PENALTY METHOD FOR THE COMPRESSIBLE NAVIER-STOKES EQUATIONS: I. OPEN BOUNDARY CONDITIONS*
}

\section{J. S. HESTHAVEN ${ }^{\dagger \ddagger}$ AND D. GOTTLIEB ${ }^{\dagger \S}$}

\begin{abstract}
The purpose of this paper is to present asymptotically stable open boundary conditions for the numerical approximation of the compressible Navier-Stokes equations in three spatial dimensions. The treatment uses the conservation form of the Navier-Stokes equations and utilizes linearization and localization at the boundaries based on these variables.

The proposed boundary conditions are applied through a penalty procedure, thus ensuring correct behavior of the scheme as the Reynolds number tends to infinity. The versatility of this method is demonstrated for the problem of a compressible flow past a circular cylinder.
\end{abstract}

Key words. open boundary conditions, stable penalty methods, Navier-Stokes equations

AMS subject classifications. 65M12, 35B35, 35Q30, 76N10

1. Introduction. In the present paper, we discuss boundary conditions for dissipative, wave-dominated problems, exemplified by the Burgers equation and the three-dimensional, compressible Navier-Stokes equations given in conservation form. The emphasis is on deriving open boundary conditions that ensure the continuous problems are well posed and on devising asymptotically stable semi-discrete schemes for imposing these conditions. The boundary conditions and the semi-discrete schemes are valid even in the limit of vanishing viscosity.

When addressing exterior wave-dominated, dissipative problems, one is often forced to introduce an artificial boundary for computational reasons. This introduces the well-known problem of specifying appropriate boundary conditions at the artificial open boundary. For purely hyperbolic problems, it is well known that enforcing these boundary conditions through the characteristic variables leads to a stable approximation. However, for dissipative wave problems the procedure is considerably more complicated.

Naturally, we require that the boundary conditions lead to a well-posed, continuous problem. For wave problems of dissipative type, the problem must, in order to be compatible with weak boundary layers, remain well posed even in the limit where the dissipation vanishes and the problem becomes purely hyperbolic. In addition to this, we want the discrete approximation of the problem to be asymptotically stable and the boundary conditions to be easily implemented.

For general nonlinear problems the issues of well-posedness and asymptotic stability are very complicated and for most problems only very little is known. However, as discussed by Kreiss and Lorenz [1], we may, for a large class of operators, simplify the problem significantly if the solutions are smooth. It was shown that in this case it is sufficient to consider the questions of well-posedness and asymptotic stability for the locally linearized, constant coefficient version of the full problem.

The energy method is applied to the linearized, constant coefficient version of the continuous problem in order to obtain energy inequalities which bound the temporal growth of the solutions to the initial-boundary value problem. This technique allows the handling of such

\footnotetext{
${ }^{*}$ Received by the editors May 25, 1994; accepted for publication (in revised form) January 20, 1995.

${ }^{\dagger}$ Division of Applied Mathematics, Brown University, Providence, RI 02912 (am420000@ brownvm.bitnet).

$\ddagger$ The research of the first author was supported by Risø National Laboratory, the Danish Computing Center for Research and Education (UNI॰C), and by the Danish Science Academy.

$\S$ The research of the second author was supported by AFOSR grant 93-0090, DARPA grant N00014-91-J-4016, and NSF grant DMS-9211820.
} 
complex problems as the Navier-Stokes equations and is in general applicable to symmetrizable systems of conservation laws for which an entropy function can be defined [2].

The usual way to enforce the boundary conditions in the numerical scheme, once their proper form for the continuous problem is known, is to solve the equation in the interior of the computational domain and then enforce the boundary conditions at the boundary points. However, this approach does not take into account the fact that the equation should be obeyed arbitrarily close to the open boundary. To circumvent this problem, Funaro and Gottlieb $[3,4]$ and Carpenter, Gottlieb, and Abarbanel [5] developed the penalty method, which enforces the boundary conditions as well as considers the equation at the boundary. They showed asymptotic stability for the scheme applied to scalar hyperbolic equations and systems of hyperbolic equations. Don and Gottlieb [6] showed how this idea can help in applying the Legendre collocation method on the Chebyshev grids.

The proofs presented in this paper are all done for semi-discrete schemes. The relation between the stability of the semi-discrete and the fully discrete scheme was recently discussed by Kreiss and $\mathrm{Wu}$ [7].

The issue of well-posed boundary conditions for the compressible Navier-Stokes equations was previously considered by Gustafsson and Sundström [8], Oliger and Sundström [9], and Nordström [10,11]. They all used the energy method to derive boundary conditions for the linearized, constant coefficient Navier-Stokes equations in the primitive variable formulation. Dutt [12] introduced an entropy function, which allowed him to derive boundary conditions for the nonlinear problem, ensuring that the solution remains bounded in an entropy norm. Halpern [13] has devised well-posed artificial boundary conditions that remain valid in the limit of vanishing viscosity. He approached the problem by viewing the linearized Navier-Stokes equations as a hyperbolic system subjected to an incomplete elliptic perturbation and obtained nonlocal as well as local open boundary conditions by considering the Fourier-Laplace transformed problem in the semi-infinite half-plane. However, for most of the previous work only few suggestions are given on how to enforce the derived boundary conditions in a natural and consistent way.

The remaining part of this paper is organized as follows. In $\S 2$ we review some well-known results on Legendre polynomials and collocation methods. Section 3 discusses the Burgers equation and boundary conditions that ensure well-posedness of the problem are derived. We continue by proposing an asymptotically stable penalty method through which the boundary conditions are enforced. This scheme ensures the correct behavior even in the limit, where the problem becomes hyperbolic, and may in general be applied to any nonlinear scalar equation. The penalty method for linear scalar hyperbolic, parabolic, and advection-diffusion equations is briefly discussed and the proposed scheme is evaluated by numerical tests. The importance of properly choosing the penalty parameter is addressed in $\S 4$, where we discuss the effect of the penalty method on the Courant-Friedrichs-Levy (CFL) condition when using explicit RungeKutta methods for time-stepping linear problems. We show that the results from the linear analysis carry over to the nonlinear case by performing simulations of the Burgers equation. We briefly discuss the equivalent penalty method for Chebyshev collocation methods. In $\S 5$ we derive open boundary conditions for the compressible Navier-Stokes equations given in conservation form and propose a penalty method for enforcing these boundary conditions. We derive the symmetrized form of the Navier-Stokes equation in conservation form and prove well-posedness for the continuous case and asymptotic stability of the proposed semidiscrete scheme using a Legendre collocation method. The boundary conditions and the semi-discrete approximation remain valid in the limit where the Reynolds number approaches infinity. The performance of the scheme in direct simulations of the Navier-Stokes equations is illustrated by simulating compressible flow around a circular cylinder using a Fourier- 
Chebyshev collocation method. Section 6 concludes the paper and discusses the application of the scheme in finite difference/finite element simulations.

2. Legendre polynomials and collocation methods. The schemes, which we analyze in the present paper, are all based on Legendre collocation methods. This choice is dictated merely by a wish to obtain analytical results, and the methods extend trivially to other collocation methods and even to finite difference/finite element methods.

The Legendre polynomial of order $N, P_{N}(x)$, is defined as

$$
P_{N}(x)=\frac{1}{2^{N} N !} \frac{d^{N}}{d x^{N}}\left(x^{2}-1\right)^{N},
$$

where $|x| \leq 1$. In what follows, we will only consider collocation methods where the collocation points are given as the Legendre-Gauss-Lobatto points, defined as the roots of the polynomial $\left(1-x^{2}\right) P_{N}^{\prime}(x)$. There is no known explicit formula for these roots.

Associated with the Gauss-Lobatto points is the quadrature formula, stating that if $f(x)$ is a polynomial of degree $2 N-1$, then

$$
\sum_{k=0}^{N} f\left(x_{k}\right) \omega_{k}=\int_{-1}^{1} f(\xi) d \xi
$$

where $x_{k}$ are the Legendre-Gauss-Lobatto collocation points, and the Gauss-Lobatto weights, $\omega_{k}$, are given as

$$
\begin{aligned}
& \omega_{k}=-\frac{2}{N+1} \frac{1}{P_{N}\left(x_{k}\right) P_{N-1}^{\prime}\left(x_{k}\right)} \quad, 1 \leq k \leq N-1, \\
& \omega_{0}=\omega_{N}=\frac{2}{N(N+1)} .
\end{aligned}
$$

For further details on the properties of the Legendre polynomials, we refer to [14].

In a Legendre collocation method, the function, $f(x)$, is approximated by a grid function, $f_{k}=f\left(x_{k}\right)$, where the grid points are the Gauss-Lobatto collocation points. Thus, we construct a global Legendre interpolant, $I_{N}$, to obtain an approximation to the function as

$$
\left(I_{N} f\right)(x)=\sum_{k=0}^{N} f_{k} h_{k}(x)
$$

where the interpolating Legendre-Lagrange polynomials are given as

$$
h_{k}(x)=-\frac{\left(1-x^{2}\right) P_{N}^{\prime}(x)}{N(N+1)\left(x-x_{k}\right) P_{N}\left(x_{k}\right)} .
$$

We note that by construction,

$$
\left(I_{N} f\right)\left(x_{k}\right)=f_{k}
$$

To seek equations for an approximate solution, $\left(I_{N} f\right)(x)$, to a partial differential equation, we need to obtain values for the spatial derivatives at the collocation points. This is done by approximating the differential operator by a matrix operator, with the matrix entries given as

$$
\mathcal{D}_{k l}=h_{l}^{\prime}\left(x_{k}\right) .
$$

For the explicit expression of the entries, we refer to $[15,16]$. 
3. The Burgers equation. In this section, we consider the Burgers equation

$$
\frac{\partial U}{\partial t}+U \frac{\partial U}{\partial x}=\varepsilon \frac{\partial^{2} U}{\partial x^{2}} \quad|x| \leq 1 \quad t>0,
$$

where $\varepsilon \geq 0$. The initial condition is given as

$$
U(x, 0)=f(x),
$$

with boundary conditions of the form

$$
\begin{aligned}
& \alpha U(-1, t)-\left.\beta \varepsilon \frac{\partial U}{\partial x}\right|_{x=-1}=0, \\
& \gamma U(1, t)+\left.\delta \varepsilon \frac{\partial U}{\partial x}\right|_{x=1}=0 .
\end{aligned}
$$

When addressing the issue of well-posedness it is sufficient to consider the linearized, constant coefficient version of the Burgers equation

$$
\frac{\partial U}{\partial t}+\lambda \frac{\partial U}{\partial x}=\varepsilon \frac{\partial^{2} U}{\partial x^{2}} \quad|x| \leq 1 t>0 .
$$

Here $\lambda=U_{0}$ is the uniform solution around which we have linearized. Equation (6) is also known as the linear advection-diffusion equation.

The four real constants, $\alpha, \beta, \gamma$, and $\delta$, in the boundary conditions, (4) and (5), may not be chosen arbitrarily, since the resulting problem should be well posed. Bounds yielding a sufficient condition for well-posedness are given in the following lemma.

LEMMA 3.1. Equation (6), with boundary conditions given by (4) and (5), is well posed if one of the following conditions holds:

(i) $\beta=0, \delta=0$.

(ii) $\beta \neq 0, \delta=0$ and $(\varepsilon-\lambda)+2 \alpha / \beta \geq 0$.

(iii) $\beta=0, \delta \neq 0$ and $(\varepsilon+\lambda)+2 \gamma / \delta \geq 0$.

(iv) $\beta \neq 0, \delta \neq 0$ and $2(\varepsilon-\lambda) \gamma / \delta+2(\varepsilon+\lambda) \alpha / \beta+4(\alpha \gamma) /(\beta \delta) \geq \lambda^{2}$.

Proof. Construct the energy integral as

$$
\frac{1}{2} \frac{d}{d t}\|U\|^{2}=-\lambda\left(U, U_{x}\right)+\varepsilon\left(U, U_{x x}\right)=\frac{1}{2}\left[-\lambda U^{2}+2 \varepsilon U U_{x}\right]_{-1}^{1}-\varepsilon\left\|U_{x}\right\|^{2} .
$$

Here we have introduced

$$
(U, V)=\int_{-1}^{1} U V d x \quad, \quad(U, U)=\|U\|^{2} .
$$

Following an analysis similar to that in [17], we apply the following

$$
-\varepsilon\left\|U_{x}\right\|^{2} \leq-\frac{\varepsilon}{2}[U(1)-U(-1)]^{2} .
$$

Following this, the condition for well-posedness becomes

$$
\frac{1}{2} \frac{d}{d t}\|U\|^{2} \leq \frac{1}{2}\left[-\lambda U^{2}+2 \varepsilon U U_{x}\right]_{-1}^{1}-\frac{\varepsilon}{2}[U(1)-U(-1)]^{2} \leq 0 .
$$

Condition (i) implies that $U(-1)=U(1)=0$ such that

$$
\frac{1}{2} \frac{d}{d t}\|U\|^{2} \leq 0 \text {. }
$$


For condition (ii) we obtain $U(1)=0$ and thus

$$
\frac{1}{2} \frac{d}{d t}\|U\|^{2} \leq-\frac{1}{2}\left(\varepsilon-\lambda+2 \frac{\alpha}{\beta}\right) U^{2}(-1) \leq 0,
$$

yielding the condition

$$
\varepsilon-\lambda+2 \frac{\alpha}{\beta} \geq 0
$$

Likewise, for condition (iii) we obtain

$$
\frac{1}{2} \frac{d}{d t}\|U\|^{2} \leq-\frac{1}{2}\left(\varepsilon+\lambda+2 \frac{\gamma}{\delta}\right) U^{2}(1) \leq 0,
$$

showing that this choice yields well-posedness. For condition (iv) we obtain the constraint

$$
\frac{1}{2} \frac{d}{d t}\|U\|^{2} \leq-\frac{1}{2}\left(\varepsilon-\lambda+2 \frac{\alpha}{\beta}\right) U^{2}(-1)+\varepsilon U(-1) U(1)-\frac{1}{2}\left(\varepsilon+\lambda+2 \frac{\gamma}{\delta}\right) U^{2}(1) \leq 0 .
$$

This is obeyed if

$$
\varepsilon^{2}-\left(\varepsilon-\lambda+2 \frac{\alpha}{\beta}\right)\left(\varepsilon+\lambda+2 \frac{\gamma}{\delta}\right) \leq 0
$$

implying

$$
2(\varepsilon-\lambda) \gamma / \delta+2(\varepsilon+\lambda) \alpha / \beta+4(\alpha \gamma) /(\beta \delta) \geq \lambda^{2} .
$$

3.1. The semi-discrete scheme. Equation (3) will be solved using a Legendre collocation method where the collocation points are the Legendre-Gauss-Lobatto points. This involves finding an $N$ th degree polynomial, $u(x, t)$, satisfying

$$
\frac{\partial u}{\partial t}+u \frac{\partial u}{\partial x}=\varepsilon \frac{\partial^{2} u}{\partial x^{2}} \text { at } x=x_{k}, k \in[1 \ldots N-1],
$$

in the interior. The boundary points are given by boundary conditions of Robin type

$$
\begin{aligned}
& \alpha u\left(x_{0}, t\right)-\left.\beta \varepsilon \frac{\partial u}{\partial x}\right|_{x_{0}}=g_{1}(t), \\
& \gamma u\left(x_{N}, t\right)+\left.\delta \varepsilon \frac{\partial u}{\partial x}\right|_{x_{N}}=g_{2}(t),
\end{aligned}
$$

where $g_{1}(t)$ and $g_{2}(t)$ are prescribed boundary conditions. The traditional method of imposing the boundary conditions is to solve (7) in the interior and enforce the boundary conditions at the boundary points only. However, this approach does not take into account the fact that the equation must be obeyed arbitrarily close to the boundary. In addition to this, it has proven difficult to implement Robin boundary conditions consistently when using spectral approximations of nonlinear problems. To overcome these problems, we follow the line of thought initiated by Funaro and Gottlieb [3, 4] and propose a penalty method for approximating the Burgers equation at the Legendre-Gauss-Lobatto collocation points, $x=x_{k}, k \in[0, \ldots, N]$, as

$$
\begin{aligned}
\frac{\partial u}{\partial t}+u \frac{\partial u}{\partial x}= & \varepsilon \frac{\partial^{2} u}{\partial x^{2}} \\
& -\tau_{1} Q^{-}(x)\left[\alpha u\left(x_{0}, t\right)-\left.\beta \varepsilon \frac{\partial u}{\partial x}\right|_{x_{0}}-g_{1}(t)\right] \\
& -\tau_{2} Q^{+}(x)\left[\gamma u\left(x_{N}, t\right)+\left.\delta \varepsilon \frac{\partial u}{\partial x}\right|_{x_{N}}-g_{2}(t)\right],
\end{aligned}
$$


where

$$
Q^{-}(x)=\frac{(1-x) P_{N}^{\prime}(x)}{2 P_{N}^{\prime}(-1)}, Q^{+}(x)=\frac{(1+x) P_{N}^{\prime}(x)}{2 P_{N}^{\prime}(1)} .
$$

These two functions have the property of being zero at all the Legendre-Gauss-Lobatto collocation points except at the two endpoints of the domain. Although $Q^{-}$and $Q^{+}$here are defined as delta-functions at the boundary, we may also choose other definitions. As shown by Don and Gottlieb [6], this approach may also be applied for implementing Legendre methods at Chebyshev grids.

We note here that the penalty method as given by (8) combines the boundary conditions and the governing equation into one equation. When using the penalty method, the boundary conditions are only enforced weakly at the boundary. However, the method remains spectrally accurate, as we will soon illustrate. One may also observe that the scheme is equivalent to the traditional way of imposing boundary conditions as $\tau_{1}, \tau_{2}$ approach infinity.

The parameters, $\tau_{1}$ and $\tau_{2}$, are then to be determined such that the semi-discrete approximation to the initial-boundary value problem is asymptotically stable.

In order to obtain the energy inequality, we consider only homogeneous boundary conditions. As discussed in [1], this is not a restriction, since we may always introduce a variable transform such the boundary conditions become homogeneous. In the following lemma we state the bounds on $\tau_{1}$ and $\tau_{2}$ which ensure that the linearized, constant coefficient version of (8) is asymptotically stable.

LEMMA 3.2. Assume $u(x, t)$ exists and let $\tau_{a, b}^{-}$and $\tau_{a, b}^{+}$be defined as

$$
\begin{aligned}
& \tau_{a, b}^{-}=\frac{1}{\omega \varepsilon b}\left[\varepsilon+2 \kappa-2 \sqrt{\kappa^{2}+\varepsilon \kappa-1 / 2 \varepsilon \omega|\lambda|}\right], \\
& \tau_{a, b}^{+}=\frac{1}{\omega \varepsilon b}\left[\varepsilon+2 \kappa+2 \sqrt{\kappa^{2}+\varepsilon \kappa-1 / 2 \varepsilon \omega|\lambda|}\right],
\end{aligned}
$$

where $\kappa=\omega a / b$ and

$$
\omega=\frac{2}{N(N+1)}
$$

is the Legendre weight at the endpoints.

If

$$
\begin{aligned}
& \tau_{\alpha, \beta}^{-} \leq \tau_{1} \leq \tau_{\alpha, \beta}^{+}, \\
& \tau_{\gamma, \delta}^{-} \leq \tau_{2} \leq \tau_{\gamma, \delta}^{+}
\end{aligned},
$$

then the linearized, constant coefficient version of $(8)$ is asymptotically stable and the solution is bounded as

$$
\frac{1}{2} \frac{d}{d t}\|u\|_{N}^{2} \leq-\varepsilon \sum_{k=1}^{N-1}\left(\frac{\partial u}{\partial x}\left(x_{k}\right)\right)^{2} \omega_{k} .
$$

Proof. We start by defining the discrete, weighted inner product as

$$
(u, v)_{N}=\sum_{k=0}^{N} u\left(x_{k}\right) v\left(x_{k}\right) \omega_{k},(u, u)_{N}=\|u\|_{N}^{2}
$$

and note that since we are using a Legendre collocation method, we have, through (1), the identity

$$
\left(u, v_{x}\right)_{N}=\left(U, V_{x}\right)
$$


This makes it straightforward to apply partial differentiation. Following the results stated previously, it is sufficient to obtain the energy estimate for the linearized, constant coefficient version of (8) with homogeneous boundary conditions:

$$
\begin{aligned}
\frac{1}{2} \frac{d}{d t}\|u\|_{N}^{2}= & -\frac{\lambda}{2}\left[u^{2}\right]_{-1}^{1}+\varepsilon\left[u u_{x}\right]_{-1}^{1}-\varepsilon\left\|u_{x}\right\|_{N}^{2} \\
& -\tau_{1} \omega u(-1)\left[\alpha u(-1)-\beta \varepsilon u_{x}(-1)\right]-\tau_{2} \omega u(1)\left[\gamma u(1)+\delta \varepsilon u_{x}(1)\right] .
\end{aligned}
$$

Here the subscripts designate differentiation and $\omega$ is the Legendre weight at the endpoints (2). Using the quadrature rule allows for rewriting as

$$
\left\|u_{x}\right\|_{N}^{2}=u_{x}^{2}(-1) \omega+u_{x}^{2}(1) \omega+\sum_{k=1}^{N-1} u_{x}^{2}\left(x_{k}\right) \omega_{k} .
$$

Contrary to the approach followed by Funaro and Gottlieb [3, 4], we recast the problem of stability into an algebraic eigenvalue problem. For the present problem, this may seem an additional complication. However, we find that for more complicated problems this approach greatly simplifies the proofs.

Isolating the terms contributing to stability at each boundary, we obtain two conditions for asymptotic stability:

$$
\mathbf{u}_{-}^{T} \mathcal{H}^{-} \mathbf{u}_{-} \leq 0, \quad \mathbf{u}_{+}^{T} \mathcal{H}^{+} \mathbf{u}_{+} \leq 0
$$

where $\mathbf{u}_{-}=\left[u(-1), u_{x}(-1)\right]^{T}, \mathbf{u}_{+}=\left[u(1), u_{x}(1)\right]^{T}$, and

$$
\begin{gathered}
\mathcal{H}^{-}=\frac{1}{2}\left[\begin{array}{cc}
\lambda-2 \alpha \omega \tau_{1} & -\varepsilon\left(1-\beta \omega \tau_{1}\right) \\
-\varepsilon\left(1-\beta \omega \tau_{1}\right) & -2 \varepsilon \omega
\end{array}\right], \\
\mathcal{H}^{+}=\frac{1}{2}\left[\begin{array}{cc}
-\lambda-2 \gamma \omega \tau_{2} & \varepsilon\left(1-\delta \omega \tau_{2}\right) \\
\varepsilon\left(1-\delta \omega \tau_{2}\right) & -2 \varepsilon \omega
\end{array}\right] .
\end{gathered}
$$

Since both matrices are symmetric, the problem is reduced to ensuring that $\mathcal{H}^{-}$and $\mathcal{H}^{+}$are negative, semi-definite. The eigenvalues of the two matrices are found to be

$$
\begin{aligned}
& \rho_{1,2}\left(\mathcal{H}^{-}\right)=\frac{1}{8}\left(-\zeta^{-} \pm \sqrt{\left(\zeta^{-}\right)^{2}+16 \varepsilon\left(\beta^{2} \omega^{2} \varepsilon \tau_{1}^{2}-2 \omega(\beta \varepsilon+2 \alpha \omega) \tau_{1}+2 \omega \lambda+\varepsilon\right)}\right) \\
& \rho_{1,2}\left(\mathcal{H}^{+}\right)=\frac{1}{8}\left(-\zeta^{+} \pm \sqrt{\left(\zeta^{+}\right)^{2}+16 \varepsilon\left(\delta^{2} \omega^{2} \varepsilon \tau_{2}^{2}-2 \omega(\delta \varepsilon+2 \gamma \omega) \tau_{2}-2 \omega \lambda+\varepsilon\right)}\right)
\end{aligned}
$$

where $\zeta^{-}=-2 \lambda+4 \omega \varepsilon+4 \alpha \omega \tau_{1}$ and $\zeta^{+}=2 \lambda+4 \omega \varepsilon+4 \gamma \omega \tau_{2}$. It is evident that negative semi-definiteness is ensured, provided $\zeta^{-}>0$ and $\zeta^{+}>0$, if

$$
\begin{aligned}
& \beta^{2} \omega^{2} \varepsilon \tau_{1}^{2}-2 \omega(\beta \varepsilon+2 \alpha \omega) \tau_{1}+2 \omega \lambda+\varepsilon \leq 0 \\
& \delta^{2} \omega^{2} \varepsilon \tau_{2}^{2}-2 \omega(\delta \varepsilon+2 \gamma \omega) \tau_{2}-2 \omega \lambda+\varepsilon \leq 0
\end{aligned}
$$

The roots of the two polynomials are

$$
\begin{aligned}
& \tau_{1}^{ \pm}=\frac{1}{\omega \varepsilon \beta}\left(\varepsilon+2 \kappa_{-} \pm 2 \sqrt{\kappa_{-}^{2}+\varepsilon \kappa_{-}-1 / 2 \varepsilon \omega \lambda}\right) \\
& \tau_{2}^{ \pm}=\frac{1}{\omega \varepsilon \delta}\left(\varepsilon+2 \kappa_{+} \pm 2 \sqrt{\kappa_{+}^{2}+\varepsilon \kappa_{+}+1 / 2 \varepsilon \omega \lambda}\right)
\end{aligned}
$$


where $\kappa_{-}=\omega \alpha / \beta$ and $\kappa_{+}=\omega \gamma / \delta$. We introduce

$$
\begin{aligned}
& \tau_{a, b}^{-}=\frac{1}{\omega \varepsilon b}\left[\varepsilon+2 \kappa-2 \sqrt{\kappa^{2}+\varepsilon \kappa-1 / 2 \varepsilon \omega|\lambda|}\right], \\
& \tau_{a, b}^{+}=\frac{1}{\omega \varepsilon b}\left[\varepsilon+2 \kappa+2 \sqrt{\kappa^{2}+\varepsilon \kappa-1 / 2 \varepsilon \omega|\lambda|}\right],
\end{aligned}
$$

where $\kappa=\omega a / b$. Since

$$
\tau_{a, b}^{-} \geq \frac{|\lambda|}{2 a \omega}+\frac{1}{4} \varepsilon \frac{1}{\omega^{2}},
$$

for $\varepsilon \ll 1$, this ensures $\zeta^{-}>0$ and $\zeta^{+}>0$.

Hence, stability is ensured for

$$
\begin{gathered}
\tau_{\alpha, \beta}^{-} \leq \tau_{1} \leq \tau_{\alpha, \beta}^{+}, \\
\tau_{\gamma, \delta}^{-} \leq \tau_{2} \leq \tau_{\gamma, \delta}^{+}
\end{gathered},
$$

with the solution being bounded as

$$
\frac{1}{2} \frac{d}{d t}\|u\|_{N}^{2} \leq-\varepsilon \sum_{k=1}^{N-1} u_{x}^{2}\left(x_{k}\right) \omega_{k} .
$$

3.1.1. Remarks on the penalty method for linear equations. The results stated in Lemma 3.2 allows us to derive the appropriate penalty parameter for a large class of linear equations. We consider the general linear advection-diffusion equation, (6), with the Robin boundary conditions given in (4) and (5). Solving this problem by a penalty method, equivalent to that given by (8), requires bounds on the penalty parameters in order to ensure stability of the scheme.

In what follows we will give these bounds for reference and will return to the numerical validation of these results in $\S 4$. Some of these results may be found in $[3,4,6]$, but are given here in a more general framework. Remember that $\omega^{-1} \sim \mathcal{O}\left(N^{2}\right)$.

Hyperbolic equations. $(\varepsilon=0$.)

1. $\lambda>0$. Well-posedness is ensured by choosing $\alpha>0$ and $\beta=\gamma=\delta=0$.

Thus, for this case we will only need bounds on $\tau_{1}$ :

$$
\tau_{\alpha, 0}^{-}=\frac{\lambda}{2 \omega \alpha}, \quad \tau_{\alpha, 0}^{+}=\infty
$$

The scheme for the hyperbolic case is stable for

$$
\infty \geq \tau_{1} \geq \frac{\lambda}{2 \omega \alpha} .
$$

2. $\lambda<0$. Well-posedness is ensured by choosing $\gamma>0$ and $\alpha=\beta=\delta=0$.

Thus, for this case we will only need bounds on $\tau_{2}$ :

$$
\tau_{\gamma, 0}^{-}=\frac{|\lambda|}{2 \omega \gamma}, \quad \tau_{\gamma, 0}^{+}=\infty
$$

The scheme for the hyperbolic case is stable for

$$
\infty \geq \tau_{2} \geq \frac{|\lambda|}{2 \omega \gamma}
$$


Parabolic equations. $(\lambda=0, \varepsilon>0$.) Necessary and sufficient conditions for wellposedness may be obtained by choosing the four parameters, $\alpha, \beta, \gamma$, and $\delta$, properly as stated in Lemma 3.1 [17]. We only state the results for the bounds of $\tau_{1}$, since the results for $\tau_{2}$ are equivalent.

1. Dirichlet boundary condition $(\alpha>0, \beta=0)$.

$$
\tau_{\alpha, 0}^{-}=\frac{\varepsilon}{4 \alpha} \frac{1}{\omega^{2}}, \quad \tau_{\alpha, 0}^{+}=\infty
$$

Stability is ensured for

$$
\infty \geq \tau_{1} \geq \frac{\varepsilon}{4 \alpha} \frac{1}{\omega^{2}}
$$

2. Neumann boundary condition $(\alpha=0, \beta>0)$.

$$
\tau_{0, \beta}^{-}=\frac{1}{\beta \omega}, \quad \tau_{0, \beta}^{+}=\frac{1}{\beta \omega} .
$$

Stability is ensured for

$$
\tau_{1}=\frac{1}{\beta \omega} .
$$

3. Robin boundary condition $(\alpha>0, \beta>0)$.

$$
\begin{aligned}
& \tau_{\alpha, \beta}^{-}=\frac{1}{\omega \varepsilon \beta}\left[\varepsilon+2 \kappa-2 \sqrt{\kappa^{2}+\varepsilon \kappa}\right], \\
& \tau_{\alpha, \beta}^{+}=\frac{1}{\omega \varepsilon \beta}\left[\varepsilon+2 \kappa+2 \sqrt{\kappa^{2}+\varepsilon \kappa}\right],
\end{aligned}
$$

where $\kappa=\omega \alpha / \beta$. Stability is ensured for

$$
\tau_{\alpha, \beta}^{+} \geq \tau_{1} \geq \tau_{\alpha, \beta}^{-} .
$$

Advection-diffusion equations. $(\lambda \neq 0, \varepsilon \geq 0)$. Again we must ensure well-posedness by proper choice of the four parameters as given by Lemma 3.1. We only state the results for the bounds of $\tau_{1}$ since the results for $\tau_{2}$ are equivalent.

1. Dirichlet boundary condition $(\alpha>0, \beta=0)$.

$$
\tau_{\alpha, 0}^{-}=\frac{|\lambda|}{2 \alpha} \frac{1}{\omega}+\frac{\varepsilon}{4 \alpha} \frac{1}{\omega^{2}}, \quad \tau_{\alpha, 0}^{+}=\infty
$$

Stability is ensured for

$$
\infty \geq \tau_{1} \geq \frac{|\lambda|}{2 \alpha} \frac{1}{\omega}+\frac{\varepsilon}{4 \alpha} \frac{1}{\omega^{2}} .
$$

2. Neumann boundary condition $(\alpha=0, \beta>0)$.

$$
\tau_{0, \beta}^{-}=\frac{1}{\beta \omega}-\sqrt{\frac{2|\lambda| \omega}{\epsilon \beta^{2}}}, \quad \tau_{0, \beta}^{+}=\frac{1}{\beta \omega}+\sqrt{\frac{2|\lambda| \omega}{\epsilon \beta^{2}}} .
$$

Stability is ensured for

$$
\frac{1}{\beta \omega}+\sqrt{\frac{2|\lambda| \omega}{\epsilon \beta^{2}}} \geq \tau_{1} \geq \frac{1}{\beta \omega}-\sqrt{\frac{2|\lambda| \omega}{\epsilon \beta^{2}}} .
$$

3. Robin boundary conditions, $\alpha>0, \beta>0$. Results are given in Lemma 3.2. 
3.2. Numerical tests. Because we aim to solve the full nonlinear Burgers equation, and not the linearized, constant coefficient version, we need to validate the results obtained from the linear analysis. We have solved the Burgers equation using the scheme given by (8) and employ a standard Legendre collocation method as described in $\S 2[15,18]$. form

The Burgers equation, (3), has a rightward traveling wave solution (see, e.g., [1]) of the

$$
U(x, t)=-a \tanh \left(a \frac{x-c t}{2 \varepsilon}\right)+c, x \in[-\infty, \infty], t \geq 0,
$$

where the free-stream values

$$
\lim _{x \rightarrow-\infty} U(x, t)=b_{-\infty}, \lim _{x \rightarrow \infty} U(x, t)=b_{\infty},
$$

are associated with the wave-speed, $c$, and the constant, $a \geq 0$, as

$$
c=\frac{b_{-\infty}+b_{\infty}}{2}, a=\frac{b_{-\infty}-b_{\infty}}{2} .
$$

Following the results in Lemma 3.1 (condition (iv): $\alpha=\lambda, \beta=1, \gamma=0, \delta=1$ ), we expect the nonlinear problem to be well posed for boundary conditions of the type

$$
\lambda U(-1, t)-\varepsilon \frac{\partial U(-1, t)}{\partial x}=g_{1}(t), \quad \varepsilon \frac{\partial U(1, t)}{\partial x}=g_{2}(t),
$$

where $\lambda \geq 0$ is the value around which we have linearized locally. In the present study we have used the free-stream value at the inflow, i.e., $\lambda=b_{-\infty}$. Since we know an exact solution, the boundary conditions may be given exactly at all times using (10). As the initial condition we use

$$
U(x, 0)=-a \tanh \left(a \frac{x}{2 \varepsilon}\right)+c .
$$

The solution is time-stepped using a classical fourth-order Runge-Kutta method, where the boundary conditions are imposed at the intermediate time levels.

Using the values of the penalty parameters given in Lemma 3.2 results in a stable scheme. However, the CFL number, relating the maximum allowable time-step to the spatial resolution as

$$
\Delta t_{\max } \leq \mathrm{CFL} \times \min _{k}\left[\frac{\left|u\left(x_{k}\right)\right|}{\Delta_{k} x}+\frac{\varepsilon}{\left(\Delta_{k} x\right)^{2}}\right]^{-1},
$$

will have to be very small in order to ensure stability. Here $\left|u\left(x_{k}\right)\right|$ signifies the local absolute value of $u$ and $\Delta_{k} x$ represents the local grid spacing. Thus, with the theoretical value of the penalty parameter, the proposed method compares unfavorably with the traditional method because of severe time-step restrictions. Fortunately, the limits of the penalty parameters, in between which asymptotic stability is ensured, are obtained as a result of a conservative energy estimate and, hence, are not strict bounds.

We have used the values of the penalty parameter (see Lemma 3.2) as

$$
\tau_{1}=\frac{\tau_{\lambda, 1}^{-}}{4} \quad, \quad \tau_{2}=\frac{\tau_{0,1}^{-}}{4}
$$

These values are found to lead to a stable scheme, provided the cell Reynolds number $\operatorname{Re}_{N}=\lambda /\left(\varepsilon N^{2}\right) \ll 1$. The constraint simply states that increasing the Reynolds number 


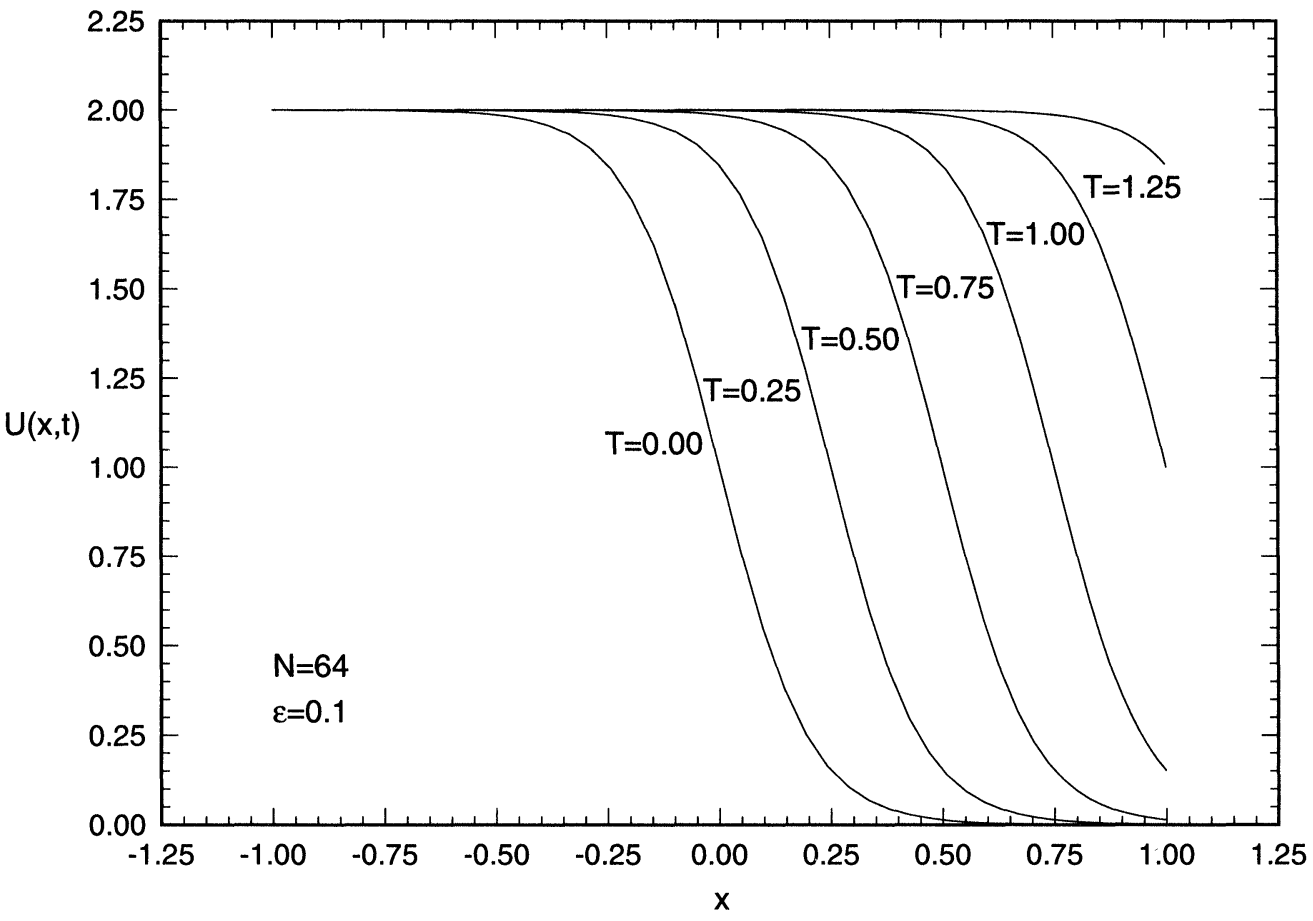

FIG. 1. Traveling wave solution of the Burgers equation.

TABLE 1

Error in the spectral simulation of the Burgers equation using the penalty method. The maximum error $\left(L_{\infty}\right)$ occurs at the boundary.

\begin{tabular}{||r||c|c||}
\hline $\mathrm{N}$ & $L_{2}$ & $L_{\infty}$ \\
\hline 16 & $3.41 \mathrm{E}-03$ & $3.26 \mathrm{E}-02$ \\
32 & $2.43 \mathrm{E}-05$ & $3.50 \mathrm{E}-04$ \\
64 & $1.09 \mathrm{E}-09$ & $2.21 \mathrm{E}-08$ \\
128 & $4.98 \mathrm{E}-12$ & $7.62 \mathrm{E}-11$ \\
\hline
\end{tabular}

requires increased spatial resolution, which is a natural restriction. For advection-dominated problems, stability is obtained by increasing the penalty parameters toward the values stated in Lemma 3.2.

With these values of the penalty parameters, we have been able to perform the simulations with a CFL number of 4 , which is equivalent to what is usually allowed when using a traditional method. Thus, by fine-tuning the penalty parameters we were able to avoid any effect of the penalty method on the CFL condition. The following section contains a study of the effect of the penalty method on the CFL condition and guidelines for fine-tuning the penalty parameter for practical applications.

In Fig. 1 we show the temporal evolution of the traveling wave solution when using the scheme given by (8). The simulation is done with $N=64$ and $\varepsilon=0.1$. We observe no spurious reflections from the open boundary and the kink is seen to travel undisturbed out of the domain. Table 1 shows the error at $T=1.00$, where the kink has propagated halfway through the boundary. It is evident that the proposed scheme maintains the spectral accuracy. The time-step is small enough to neglect time-stepping errors. 
4. CFL restrictions for the penalty method. As discussed briefly in the previous section, choosing too large a penalty parameter results in severe CFL restrictions. For this reason, it is vital to understand how the penalty method alters the eigenvalue spectrum of the operators and consequently changes the CFL restriction.

In the present section we will study these effects for the linear advection and diffusion operators for Legendre collocation methods. For completeness, we will also give the results for Chebyshev collocation methods, which are widely used for solving nonlinear partial differential equations. The analysis will include both third- and fourth-order Runge-Kutta methods, which are often employed when addressing problems of the type considered here. At the end of the section we will compare the results from our linear analysis with simulations of the nonlinear Burgers equation.

Consider now the semi-discrete linear, constant coefficient problem

$$
\begin{array}{ll}
(\mathbf{q})_{t}=\mathcal{L}_{N} \mathbf{q} & x_{k} \in \Omega, t \geq 0, \\
\mathbf{q}=0 & x_{k} \in \Omega, t=0, \\
\mathcal{B}_{N} \mathbf{q}=0 \quad x_{k} \in \Gamma, t \geq 0,
\end{array}
$$

where $\mathbf{q}=\left(\mathbf{q}\left(x_{0}\right), \ldots, \mathbf{q}\left(x_{N}\right)\right)^{T}, \mathcal{L}_{N}$ is the discrete approximation of the continuous operator for the interior, $\mathcal{L}$, and $\mathcal{B}_{N}$ determines the appropriate discrete boundary conditions by approximating the boundary operator, $\mathcal{B}$. We assume that the semi-discrete approximation is a consistent approximation of the continuous problem. A time-differencing scheme, where the boundary conditions are enforced exactly at the boundary points, may then be expressed as

$$
\begin{aligned}
& \mathbf{q}^{n+1}=K_{N}\left(\Delta t, \mathcal{L}_{N}\right) \mathbf{q}^{n}, \\
& \mathcal{B}_{N} \mathbf{q}^{n+1}=0 .
\end{aligned}
$$

Here $\mathbf{q}^{n}$ signifies the solution vector at time-step $n$. Thus, for strong stability we must require

$$
\left|K_{N}\left(\Delta t, \mathcal{L}_{N}\right)\right|<1 .
$$

However, employing the penalty method changes the time-stepping scheme to be

$$
\mathbf{q}^{n+1}=K_{N}\left(\Delta t, \mathcal{L}_{N}-\tau \mathcal{B}_{N}\right) \mathbf{q}^{n},
$$

and strong stability is ensured if

$$
\left|K_{N}\left(\Delta t, \mathcal{L}_{N}-\tau \mathcal{B}_{N}\right)\right|<1,
$$

explaining why the CFL condition depends strongly on the correct choice of the penalty parameter.

In the following analysis we consider explicit Runge-Kutta time-stepping methods, which, for time independent operators, may be expressed as

$$
K_{N}^{p}\left(\Delta t, \mathcal{L}_{N}\right)=\sum_{i=0}^{p} \frac{1}{i !}\left(\Delta t \mathcal{L}_{N}\right)^{i}
$$

where $p$ is the order of the scheme. We have for simplicity assumed that the boundary conditions are included in the operators. Assuming $\mathcal{L}_{N}=\mathcal{S}_{N} \Lambda_{N} \mathcal{S}_{N}^{-1}$, where $\left|S_{N}\right|$ and $\left|S_{N}^{-1}\right|$ are bounded independently of $N$, strong stability of the Runge-Kutta schemes is obtained if

$$
\left|K_{N}^{p}\left(\Delta t, \mathcal{L}_{N}\right)\right|=\mathcal{S}_{N}\left|\sum_{i=0}^{p} \frac{1}{i !}\left(\Delta t \Lambda_{N}\right)^{i}\right| \mathcal{S}_{N}^{-1}=\left|\sum_{i=0}^{p} \frac{1}{i !}\left(\Delta t \Lambda_{N}\right)^{i}\right|<1 .
$$


TABLE 2

Scaling constants for the advection operator. The proper boundary conditions are of Dirichlet type (D).

\begin{tabular}{|c|c|c|c|c|c|}
\hline \multirow{2}{*}{\multicolumn{2}{|c|}{$\begin{array}{l}\text { Advection Operator } \\
\lambda \neq 0 \quad \varepsilon=0\end{array}$}} & \multicolumn{2}{|c|}{$\overline{C_{L}}$} & \multicolumn{2}{|c|}{$C_{C}$} \\
\hline & & 3rd RK & 4th RK & 3rd RK & 4th RK \\
\hline $\bar{D}$ & Exact BC & 21 & 35 & 27 & 32 \\
\hline & Penalty BC & 10 & 17 & 10 & 11 \\
\hline
\end{tabular}

TABLE 3

Scaling constants for the diffusion operator. Results are given for possible combinations of Dirichlet (D), Neumann $(N)$, and Robin $(R)$ boundary conditions.

\begin{tabular}{||ll||r|r||r|r||}
\hline \multicolumn{1}{||l||}{ Diffusion Operator } & \multicolumn{2}{c||}{$C_{L}$} & \multicolumn{2}{c||}{$C_{C}$} \\
$\lambda=0 \quad \varepsilon>0$ & & 3rd RK & 4th RK & 3rd RK & 4th RK \\
\hline \multirow{2}{*}{ D-D/D-N/D-R } & Exact BC & 99 & 109 & 53 & 58 \\
& Penalty BC & 81 & 123 & 56 & 84 \\
\hline N-R & Exact BC & 99 & 109 & 53 & 58 \\
& Penalty BC & 130 & 135 & 91 & 96 \\
\hline R-R & Exact BC & 99 & 109 & 53 & 58 \\
& Penalty BC & 130 & 141 & 93 & 97 \\
\hline
\end{tabular}

Hence, the problem is reduced to finding the eigenvalue spectrum of the operator $\mathcal{L}_{N}$ and choosing $\Delta t$ accordingly.

In the present study we consider the linear advection-diffusion operator

$$
\mathcal{L}=\lambda \frac{\partial}{\partial x}+\varepsilon \frac{\partial^{2}}{\partial x^{2}}
$$

with the Robin boundary condition operators

$$
\mathcal{B}^{-}=\alpha-\varepsilon \beta \frac{\partial}{\partial x}, \mathcal{B}^{+}=\gamma+\varepsilon \delta \frac{\partial}{\partial x} .
$$

The boundary conditions for the exact method are enforced through the operator as described in [18].

In order to compare time-step restrictions found for the two different approaches, we now define the two CFL-like constants, $C_{L}$ and $C_{C}$, as

$$
\Delta t_{L} \leq \frac{C_{L}}{\lambda N(N+1)+\varepsilon N^{2}(N+1)^{2}}, \quad \Delta t_{C} \leq \frac{C_{C}}{\lambda N^{2}+\varepsilon N^{4}},
$$

where the subscripts refer to Legendre (L) and Chebyshev (C) operators, respectively. These constants are determined by solving the eigenvalue problem and calculating the maximum $\Delta t$ which ensures stability and, hence, supplies an upper bound on the time-step.

Tables 2 and 3 show the calculated values of $C_{L}$ and $C_{C}$ for the advection and the diffusion operator. The results are the same for the full advection-diffusion operator as for the diffusion operator, provided $\operatorname{Re}_{N} \ll 1$, and are therefore omitted.

It is clear from Table 2 that using the penalty method to enforce boundary conditions on purely advective problems results in a significant reduction of the maximum allowable time-step. However, more importantly, Table 3 shows that for problems where the diffusion operator dominates the eigenvalue spectrum, the penalty method may allow for increasing the time-step as much as $50 \%$. The effect is most pronounced when using a fourth-order Runge-Kutta method for time-stepping a Chebyshev collocation scheme.

In order to explain the results in Tables 2 and 3, we compare in Fig. 2 the spectrum of the Legendre collocation advection (Fig. 2a) and diffusion (Fig. 2b) operators when enforcing Dirichlet boundary conditions through the exact method and the penalty method. 


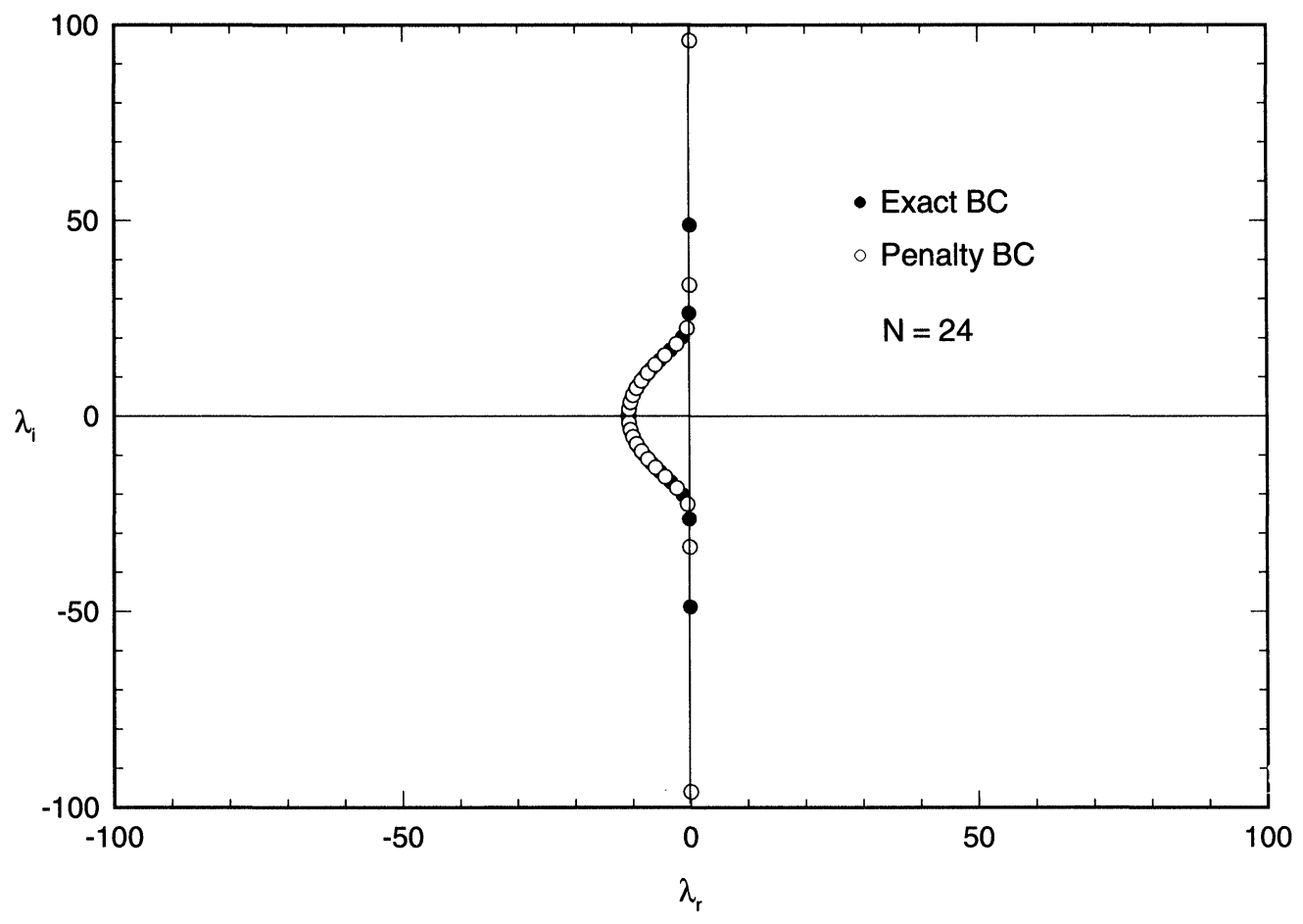

(a)

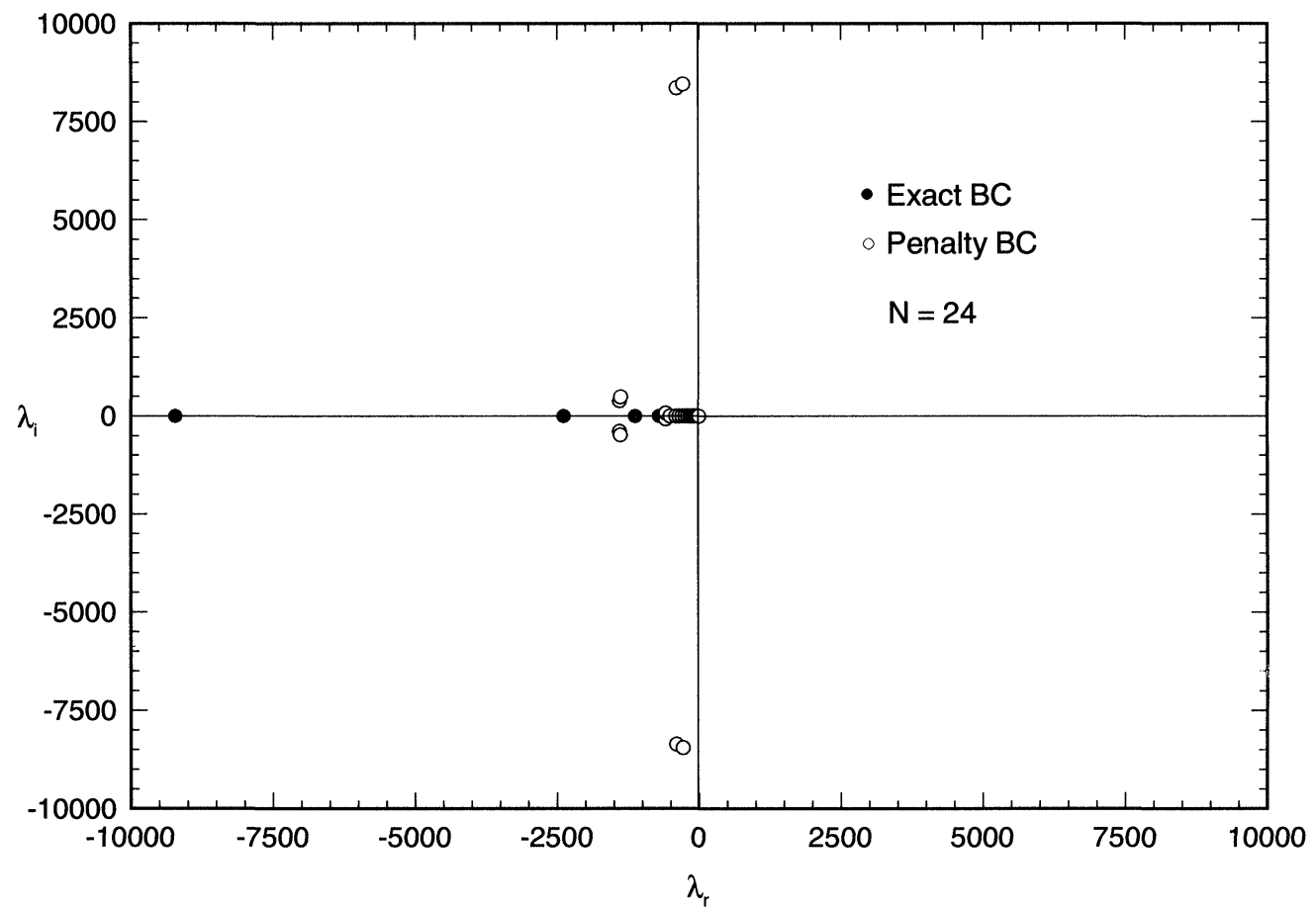

(b)

FIG. 2. Eigenvalue spectrum $\left(\lambda=\lambda_{r}+i \lambda_{i}\right)$ for the Legendre advection operator (a) and the Legendre diffusion operator (b) as obtained by using exact boundary conditions $(\bullet)$ and the penalty method $(0)$. 
For the advection operator (Fig. 2a) we observe that the effect of the penalty method is to introduce an extreme, purely imaginary, complex conjugate eigenvalue-pair, which dominates the spectrum and consequently determines the maximum allowable time-step. This results in the decreased CFL number observed in Table 2.

The effect on the diffusion operator is more complicated and depends strongly on the value of the penalty parameter. As proved by Gottlieb and Lustman [17], the diffusion operator with exact Robin boundary conditions has a real, negative, and distinct eigenvalue spectrum. This property is preserved if a sufficiently large value of $\tau$ is used in the penalty method. However, by decreasing the penalty parameter the two dominating eigenvalues split into two pairs of complex conjugate eigenvalues, which move toward the imaginary axis as $\tau$ is decreased. In Fig. $2 \mathrm{~b}$ we show the eigenvalue spectrum for the optimal choice of $\tau$. The important observation to make is that moduli of these new eigenvalues are smaller than the original extreme negative real eigenvalue. Additionally, since the dominating eigenvalue now is complex, it clearly becomes advantageous to use the fourth-order Runge-Kutta method because of the increased extension of the stability region along the imaginary axis as compared to the third-order Runge-Kutta method. Thus, we conclude that for diffusion-dominated problems, the penalty method may allow for a significant increase in the maximum CFL number when applied in conjunction with the fourth-order Runge-Kutta method. This is true for Legendre as well as Chebyshev collocation methods.

The validity of this conclusion is, however, strongly dependent on the proper choice of the penalty parameter. The values derived in the previous section do indeed ensure asymptotic stability, but result in a significant reduction in the maximum allowable CFL number. Fortunately the limits of the penalty parameters are based on a conservative energy estimate and, consequently, are not very strict. In what follows we give the penalty parameters used to obtain the results given in Tables 2 and 3. These values result in a stable scheme as long as the problem is purely advective or $\operatorname{Re}_{N} \ll 1$, and allow in most cases for a significant increase in the time-step.

\section{Legendre collocation methods.}

1. Dirichlet boundary conditions.

$$
\tau=\frac{|\lambda|}{4} N(N+1)+\frac{\varepsilon}{64} N^{2}(N+1)^{2} .
$$

2. Neumann boundary conditions.

$$
\tau=\frac{N(N+1)}{8} .
$$

3. Robin boundary conditions.

$$
\tau=\frac{\tau_{\alpha, \beta}^{-}}{4}
$$

\section{Chebyshev collocation methods.}

1. Dirichlet boundary conditions.

$$
\tau=\frac{|\lambda|}{2} N^{2}+\frac{\varepsilon}{50} N^{4} .
$$

2. Neumann boundary conditions.

$$
\tau=\frac{N^{2}}{8} .
$$




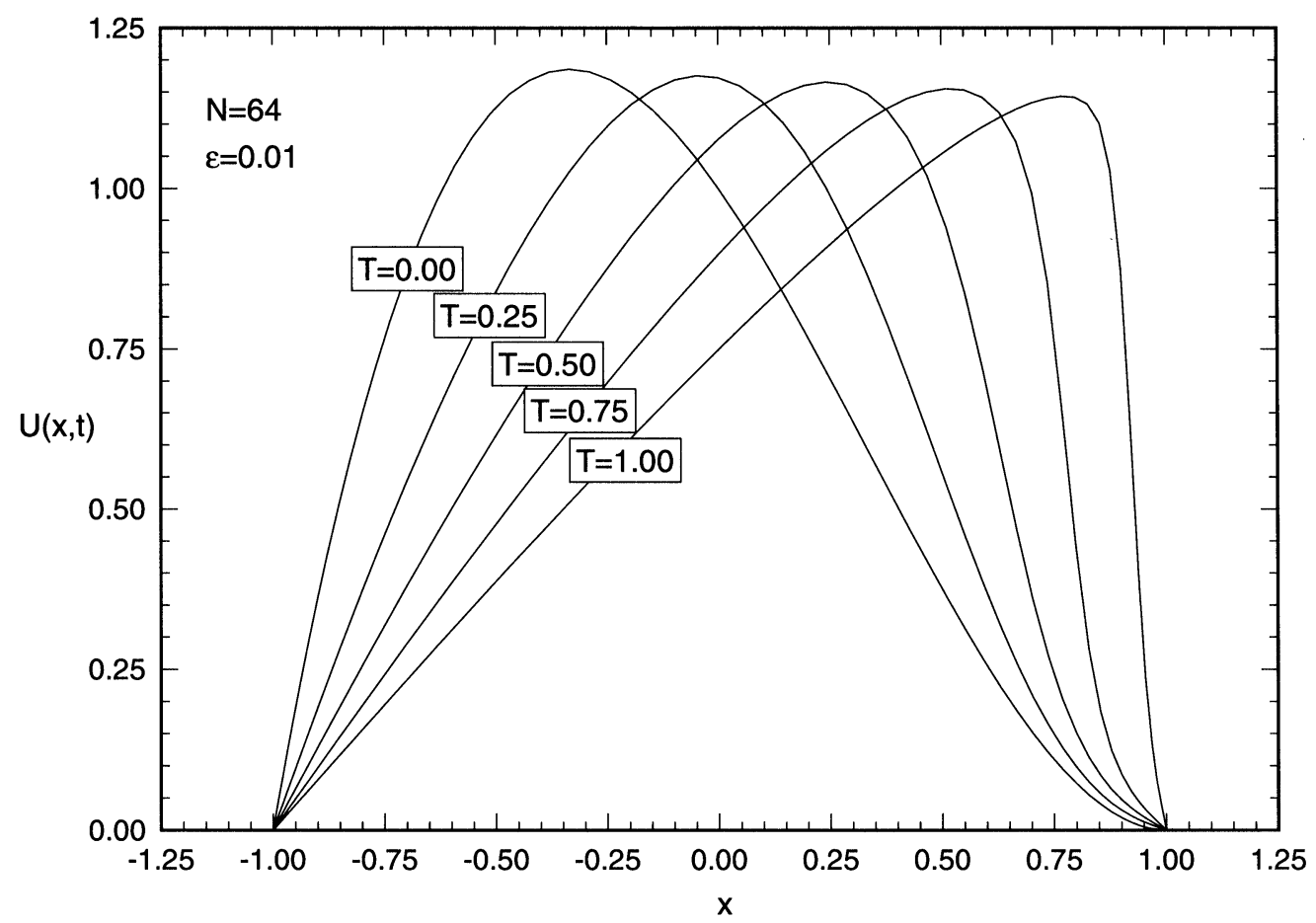

FIG. 3. Temporal evolution of the Burgers equation with initial conditions given by (12).

3. Robin boundary conditions.

$$
\tau=\frac{\tau_{\alpha, \beta}^{-}}{4} \text { with } \kappa=\frac{\alpha N^{2}}{\beta} .
$$

Note that the only difference between the parameter values quoted here and those found in Lemma 3.2 is a factor of $1 / 4$ on those terms related to the diffusion operator. This reduction is found to lead to optimal time-step restrictions.

We would like to stress the importance of choosing the appropriate value of the penalty parameter. It is our experience, that this is best done by deriving the theoretical value of this parameter through an analysis similar to that done in $\S 3.1$. This leads to a parameter which scales correctly with the resolution and other significant parameters. If the time-step restriction is dominated by a viscous time-scale, it is very likely that the theoretical estimate leads to severe time-step restrictions. However, the theoretical value may often be decreased considerably, and good results may be obtained after only a few tests. As we have seen for the Burgers equation, decreasing the penalty parameter four times leads to acceptable CFL restrictions. We are not aware of any systematic way to determine the optimal factor by which the theoretical value can be decreased, but it may usually be determined by trial and error through a few tests.

To conclude our study we have solved the Burgers equation, (3), with initial condition

$$
U(x, 0)=(1-x)\left(1-x^{2}\right)
$$

and homogeneous Dirichlet boundary conditions. A typical temporal evolution is shown in Fig. 3. In Table 4 we show the maximum CFL number resulting in a stable scheme. This result confirms that the results from the linear analysis carry over to the scalar nonlinear problem. 
TABLE 4

Maximum allowable CFL number obtained from direct numerical simulation of the Burgers equation.

\begin{tabular}{||l||c|c||c|c||}
\hline \multicolumn{1}{||c||}{} & \multicolumn{2}{c||}{ Legendre } & \multicolumn{2}{c||}{ Chebyshev } \\
& 3rd RK & 4th RK & 3rd RK & 4th RK \\
\hline Exact BC & 3.50 & 4.00 & 4.25 & 4.50 \\
Penalty BC & 3.00 & 4.50 & 4.75 & 6.75 \\
\hline
\end{tabular}

5. The compressible Navier-Stokes equations. In the present section, we obtain energy estimates for the solution of the three-dimensional compressible Navier-Stokes equations given in conservation form. Additionally, we derive open boundary conditions taking into account the full stress-tensor, and prove well-posedness for the continuous problem. The derivations follow the approach introduced in $[8,9]$. The main differences are that we develop the theory for the conservation form of the Navier-Stokes equations and that we include the off-diagonal terms of the stress-tensor in the full derivations. In the second part of this section we continue by showing how to apply the boundary conditions and prove asymptotic stability of the semi-discrete scheme.

Consider now the nondimensionalized, compressible Navier-Stokes equations given in conservation form

$$
\frac{\partial \mathbf{q}}{\partial t}+\frac{\partial \mathbf{F}}{\partial x}+\frac{\partial \mathbf{G}}{\partial y}+\frac{\partial \mathbf{H}}{\partial z}=\frac{1}{\operatorname{Re}_{\mathrm{ref}}}\left(\frac{\partial \mathbf{F}_{v}}{\partial x}+\frac{\partial \mathbf{G}_{v}}{\partial y}+\frac{\partial \mathbf{H}_{v}}{\partial z}\right),
$$

with $\mathbf{x} \in \Omega=[-1,1]^{3}$. The state vector, $\mathbf{q}$, and the inviscid flux vectors are given as

$$
\mathbf{q}=\left[\begin{array}{c}
\rho \\
\rho u \\
\rho v \\
\rho w \\
E
\end{array}\right], \quad \mathbf{F}=\left[\begin{array}{c}
\rho u \\
\rho u^{2}+p \\
\rho u v \\
\rho u w \\
(E+p) u
\end{array}\right], \quad \mathbf{G}=\left[\begin{array}{c}
\rho v \\
\rho u v \\
\rho v^{2}+p \\
\rho v w \\
(E+p) v
\end{array}\right], \quad \mathbf{H}=\left[\begin{array}{c}
\rho w \\
\rho u w \\
\rho v w \\
\rho w^{2}+p \\
(E+p) w
\end{array}\right] .
$$

Here $\rho$ is the density, $u, v, w$ are the three Cartesian velocity components, $E$ is the total energy, and $p$ is the pressure. In the remaining part of this paper we will use $(x, y, z)$ and $\left(x_{1}, x_{2}, x_{3}\right)$ interchangeably to denote the spatial coordinates. The total energy

$$
E=\rho\left(T+\frac{1}{2}\left(u^{2}+v^{2}+w^{2}\right)\right),
$$

and the pressure are related through the ideal gas law

$$
p=(\gamma-1) \rho T,
$$

where $T$ is the temperature field and $\gamma=c_{p} / c_{v}$ is the ratio between the heat capacities at constant pressure $\left(c_{p}\right)$ and volume $\left(c_{v}\right)$, respectively, and is assumed constant.

The viscous flux vectors are given as

$$
\begin{gathered}
\mathbf{F}_{v}=\left[\begin{array}{c}
0 \\
\tau_{x x} \\
\tau_{y x} \\
\tau_{z x} \\
\tau_{x x} u+\tau_{y x} v+\tau_{z x} w+\frac{\gamma k}{\operatorname{Pr}} \frac{\partial T}{\partial x}
\end{array}\right], \mathbf{G}_{v}=\left[\begin{array}{c}
0 \\
\tau_{x y} \\
\tau_{y y} \\
\tau_{z y} \\
\tau_{x y} u+\tau_{y y} v+\tau_{z y} w+\frac{\gamma k}{\operatorname{Pr}} \frac{\partial T}{\partial y}
\end{array}\right], \\
\mathbf{H}_{v}=\left[\begin{array}{c}
0 \\
\tau_{x z} \\
\tau_{y z} \\
\tau_{z z} \\
\tau_{x z} u+\tau_{y z} v+\tau_{z z} w+\frac{\gamma k}{\operatorname{Pr}} \frac{\partial T}{\partial z}
\end{array}\right] .
\end{gathered}
$$


Considering only Newtonian fluids, the stress-tensor elements are given as

$$
\begin{array}{ll}
\tau_{x x}=2 \mu \frac{\partial u}{\partial x}+\lambda\left(\frac{\partial u}{\partial x}+\frac{\partial v}{\partial y}+\frac{\partial w}{\partial z}\right), & \tau_{x y}=\tau_{y x}=\mu\left(\frac{\partial u}{\partial y}+\frac{\partial v}{\partial x}\right) \\
\tau_{y y}=2 \mu \frac{\partial v}{\partial y}+\lambda\left(\frac{\partial u}{\partial x}+\frac{\partial v}{\partial y}+\frac{\partial w}{\partial z}\right), & \tau_{y z}=\tau_{z y}=\mu\left(\frac{\partial w}{\partial y}+\frac{\partial v}{\partial z}\right) \\
\tau_{z z}=2 \mu \frac{\partial w}{\partial z}+\lambda\left(\frac{\partial u}{\partial x}+\frac{\partial v}{\partial y}+\frac{\partial w}{\partial z}\right), & \tau_{x z}=\tau_{z x}=\mu\left(\frac{\partial w}{\partial x}+\frac{\partial u}{\partial z}\right)
\end{array}
$$

where $\mu$ is the dynamic viscosity, $\lambda$ is the bulk viscosity, and $k$ is the coefficient of thermal conductivity.

The equations are normalized using the reference values, $u_{\text {ref }}=u_{0}, \rho_{\text {ref }}=\rho_{0}, \quad p_{\text {ref }}=$ $\rho_{0} u_{0}^{2}, T_{\text {ref }}=u_{0}^{2} / c_{v}$, and a reference length $L$, where $\left(\rho_{0}, u_{0}\right)$ is some uniform state, e.g., the ambient free-stream conditions of the flow. This gives a Reynolds number as $\operatorname{Re}=\rho_{0} u_{0} L / \mu_{0}$ and a Prandtl number as $\operatorname{Pr}=c_{p} \mu_{0} / k_{0}$. Note that the Reynolds number in (13), $\mathrm{Re}_{\text {ref }}$, based on the reference values, in general is different from Re. In the remaining part of the paper we shall refer to the latter as the Reynolds number unless clarification is deemed necessary. With this normalization we need to specify the Mach number, $M$, the Reynolds number, Re, the length scale, $L$, and a dimensional temperature, $T_{0}$.

5.1. Well-posedness and open boundary conditions for the continuous problem. Consider the linearized, constant coefficient form of (13). The viscous fluxes are split as

$$
\begin{aligned}
& \mathbf{F}_{\nu}=\mathbf{F}_{P}+\mathbf{F}_{M}^{y}+\mathbf{F}_{M}^{z}=\left[\begin{array}{c}
0 \\
(\lambda+2 \mu) \frac{\partial u}{\partial x} \\
\mu \frac{\partial v}{\partial x} \\
\mu \frac{\partial w}{\partial x} \\
(\lambda+2 \mu) u \frac{\partial u}{\partial x}+\mu v \frac{\partial v}{\partial x}+\mu w \frac{\partial w}{\partial x}+\frac{\gamma k}{\operatorname{Pr}} \frac{\partial T}{\partial x}
\end{array}\right] \\
& +\left[\begin{array}{c}
0 \\
\lambda \frac{\partial v}{\partial y} \\
\mu \frac{\partial u}{\partial y} \\
0 \\
\lambda u \frac{\partial v}{\partial y}+\mu v \frac{\partial u}{\partial y}
\end{array}\right]+\left[\begin{array}{c}
0 \\
\lambda \frac{\partial w}{\partial z} \\
0 \\
\mu \frac{\partial u}{\partial z} \\
\lambda u \frac{\partial w}{\partial z}+\mu w \frac{\partial u}{\partial z}
\end{array}\right] \text {, } \\
& \mathbf{G}_{v}=\mathbf{G}_{P}+\mathbf{G}_{M}^{x}+\mathbf{G}_{M}^{z}=\left[\begin{array}{c}
0 \\
\mu \frac{\partial u}{\partial y} \\
(\lambda+2 \mu) \frac{\partial v}{\partial y} \\
\mu \frac{\partial w}{\partial y} \\
\mu u \frac{\partial u}{\partial y}+(\lambda+2 \mu) v \frac{\partial v}{\partial y}+\mu w \frac{\partial w}{\partial y}+\frac{\gamma k}{\operatorname{Pr}} \frac{\partial T}{\partial y}
\end{array}\right] \\
& +\left[\begin{array}{c}
0 \\
\mu \frac{\partial v}{\partial x} \\
\lambda \frac{\partial u}{\partial x} \\
0 \\
\lambda v \frac{\partial u}{\partial x}+\mu u \frac{\partial v}{\partial x}
\end{array}\right]+\left[\begin{array}{c}
0 \\
0 \\
\lambda \frac{\partial w}{\partial z} \\
\mu \frac{\partial v}{\partial z} \\
\lambda v \frac{\partial w}{\partial z}+\mu w \frac{\partial v}{\partial z}
\end{array}\right]
\end{aligned}
$$




$$
\begin{aligned}
\mathbf{H}_{v}=\mathbf{H}_{P}+\mathbf{H}_{M}^{x}+\mathbf{H}_{M}^{y} & =\left[\begin{array}{c}
0 \\
\mu \frac{\partial u}{\partial z} \\
\mu \frac{\partial v}{\partial z} \\
(\lambda+2 \mu) \frac{\partial w}{\partial z} \\
\mu u \frac{\partial u}{\partial z}+\mu v \frac{\partial v}{\partial z}+(\lambda+2 \mu) w \frac{\partial w}{\partial z}+\frac{\gamma k}{\operatorname{Pr}} \frac{\partial T}{\partial z}
\end{array}\right] \\
& +\left[\begin{array}{c}
0 \\
0 \\
\mu \frac{\partial w}{\partial x} \\
0 \\
\lambda \frac{\partial u}{\partial x} \\
\lambda w \frac{\partial w}{\partial x}+\mu u \frac{\partial w}{\partial x}
\end{array}\right]+\left[\begin{array}{c}
\lambda \frac{\partial v}{\partial y} \\
\lambda w \frac{\partial v}{\partial y}+\mu v \frac{\partial w}{\partial y}
\end{array}\right] .
\end{aligned}
$$

Introducing the transformation Jacobians

$$
\begin{gathered}
\mathcal{A}_{1}=\frac{\partial \mathbf{F}}{\partial \mathbf{q}} \quad, \quad \mathcal{A}_{2}=\frac{\partial \mathbf{G}}{\partial \mathbf{q}}, \quad \mathcal{A}_{3}=\frac{\partial \mathbf{H}}{\partial \mathbf{q}}, \\
\mathcal{B}_{11}=\frac{\partial \mathbf{F}_{P}}{\partial \mathbf{q}_{x}}, \quad \mathcal{B}_{22}=\frac{\partial \mathbf{G}_{P}}{\partial \mathbf{q}_{y}}, \quad \mathcal{B}_{33}=\frac{\partial \mathbf{H}_{P}}{\partial \mathbf{q}_{z}}, \\
\mathcal{B}_{12}=\left(\frac{\partial \mathbf{F}_{M}^{y}}{\partial \mathbf{q}_{y}}+\frac{\partial \mathbf{G}_{M}^{x}}{\partial \mathbf{q}_{x}}\right), \mathcal{B}_{23}=\left(\frac{\partial \mathbf{G}_{M}^{z}}{\partial \mathbf{q}_{z}}+\frac{\partial \mathbf{H}_{M}^{y}}{\partial \mathbf{q}_{y}}\right), \mathcal{B}_{13}=\left(\frac{\partial \mathbf{F}_{M}^{z}}{\partial \mathbf{q}_{z}}+\frac{\partial \mathbf{H}_{M}^{x}}{\partial \mathbf{q}_{x}}\right),
\end{gathered}
$$

allows us to write Navier-Stokes equations as

$$
\frac{\partial \mathbf{q}}{\partial t}+\sum_{i=1}^{3} \mathcal{A}_{i} \frac{\partial \mathbf{q}}{\partial x_{i}}=\frac{1}{\operatorname{Re}_{\mathrm{ref}}} \sum_{i=1}^{3} \sum_{j=i}^{3} \mathcal{B}_{i j} \frac{\partial^{2} \mathbf{q}}{\partial x_{i} \partial x_{j}} .
$$

It is well known that the Navier-Stokes equations, although not of hyperbolic nature, support waves very similar to those encountered in the hyperbolic Euler equations. For hyperbolic systems, Gottlieb, Gunzburger, and Turkel [19] have shown that enforcing the boundary conditions through the characteristic variables of the system results in a stable approximation.

For Navier-Stokes equations, we linearize around a uniform state, $\mathbf{q}_{0}$, by fixing all the matrices. We transform into characteristic variables by diagonalizing $\mathcal{A}_{1}$ through a similarity transform $\Lambda=\mathcal{S}^{-1} \mathcal{A}_{1} \mathcal{S}$, where $\Lambda$ is the eigenvalue matrix and $\mathcal{S}$ and $\mathcal{S}^{-1}$ are the matrices of right and left eigenvectors, respectively. These matrices are given in the Appendix. Applying this, the symmetrized, linearized set of equations transforms into

$$
\mathcal{Q}^{T} \mathcal{Q} \frac{\partial \mathbf{R}}{\partial t}+\sum_{i=1}^{3} \mathcal{A}_{i}^{s} \frac{\partial \mathbf{R}}{\partial x_{i}}=\frac{1}{\operatorname{Re}_{\mathrm{ref}}} \sum_{i=1}^{3} \sum_{j=i}^{3} \mathcal{B}_{i j}^{s} \frac{\partial^{2} \mathbf{R}}{\partial x_{i} \partial x_{j}}
$$

where $\mathbf{R}=\mathcal{S}^{-1} \mathbf{q}$ are the characteristic variables. We have introduced a positive definite, symmetrizing diagonal matrix, $\mathcal{Q}^{T} \mathcal{Q}$, given as

$$
\mathcal{Q}^{T} \mathcal{Q}=\left[\begin{array}{ccccc}
1 & 0 & 0 & 0 & 0 \\
0 & 2 & 0 & 0 & 0 \\
0 & 0 & \frac{2 c_{0}^{2}}{\gamma-1} & 0 & 0 \\
0 & 0 & 0 & 2 & 0 \\
0 & 0 & 0 & 0 & 1
\end{array}\right]
$$


where $c_{0}=\sqrt{\gamma p_{0} / \rho_{0}}$ is the uniform state sound speed. Additionally we define the symmetrized matrices

$$
\mathcal{A}_{i}^{s}=\mathcal{Q}^{T} \mathcal{Q} \mathcal{S}^{-1} \mathcal{A}_{i} \mathcal{S}, \quad \mathcal{B}_{i j}^{s}=\mathcal{Q}^{T} \mathcal{Q} \mathcal{S}^{-1} \mathcal{B}_{i j} \mathcal{S}
$$

The explicit forms of the symmetric matrices are given in the Appendix. The characteristic variables, $\mathbf{R}=\left[R_{1}, R_{2}, R_{3}, R_{4}, R_{5}\right]^{T}$, are given as

$$
\mathbf{R}=\left[\begin{array}{c}
\rho u-u_{0} \rho+\frac{\gamma-1}{c_{0}}\left(E+\frac{1}{2}\left(u_{0}^{2}+v_{0}^{2}+w_{0}^{2}\right) \rho-u_{0} \rho u-v_{0} \rho v-w_{0} \rho w\right) \\
\rho v-v_{0} \rho \\
\rho-\frac{\gamma-1}{c_{0}^{2}}\left(E+\frac{1}{2} \rho\left(u_{0}^{2}+v_{0}^{2}+w_{0}^{2}\right)-u_{0} \rho u-v_{0} \rho v-w_{0} \rho w\right) \\
\rho w-w_{0} \rho \\
-\left(\rho u-u_{0} \rho\right)+\frac{\gamma-1}{c_{0}}\left(E+\frac{1}{2} \rho\left(u_{0}^{2}+v_{0}^{2}+w_{0}^{2}\right)-u_{0} \rho u-v_{0} \rho v-w_{0} \rho w\right)
\end{array}\right] .
$$

We are now ready to state the following lemma.

LEMMA 5.1. Assume there exists a solution, q, which is periodic or held at a constant value at the $y$-and $z$-boundary. If the boundary conditions in the $x$-direction are given such that

$$
\forall(y, z) \in \Omega_{y} \times \Omega_{z}:-\frac{1}{2}\left[\mathbf{R}^{T} \mathcal{A}_{1}^{s} \mathbf{R}-\frac{2}{\operatorname{Re}_{\mathrm{ref}}} \sum_{j=1}^{3} \mathbf{R}^{T} \mathcal{B}_{1 j}^{s} \frac{\partial \mathbf{R}}{\partial x_{j}}\right]_{x=-1}^{1} \leq 0,
$$

and the fluid properties are constrained by

$$
\mu_{0} \geq 0, \quad \lambda_{0} \leq 0, \quad \lambda_{0}+\mu_{0} \geq 0, \quad \frac{\gamma k_{0}}{\operatorname{Pr}} \geq 0, \quad \gamma \geq 1,
$$

then (14) constitutes a well-posed problem and the solution is bounded as

$$
\frac{1}{2} \frac{d}{d t}\|\mathcal{Q} \mathbf{R}\|^{2} \leq-\frac{1}{\operatorname{Re}_{\mathrm{ref}}} \int_{\Omega}\left(\sum_{i=1}^{3} \sum_{j=i}^{3} \frac{\partial \mathbf{R}^{T}}{\partial x_{i}} \mathcal{B}_{i j}^{s} \frac{\partial \mathbf{R}}{\partial x_{j}}\right) d \Omega \leq 0
$$

Proof. Construct the energy integral as

$$
\begin{aligned}
\frac{1}{2} \frac{d}{d t}\|\mathcal{Q R}\|^{2}= & \int_{\Omega}\left(-\sum_{i=1}^{3} \mathbf{R}^{T} \mathcal{A}_{i}^{s} \frac{\partial \mathbf{R}}{\partial x_{i}}+\frac{1}{\operatorname{Re}_{\mathrm{ref}}} \sum_{i=1}^{3} \sum_{j=i}^{3} \mathbf{R}^{T} \mathcal{B}_{i j}^{s} \frac{\partial^{2} \mathbf{R}}{\partial x_{i} \partial x_{j}}\right) d \Omega \\
= & \int_{\Omega_{y}} \int_{\Omega_{z}}-\frac{1}{2}\left[\mathbf{R}^{T} \mathcal{A}_{1}^{s} \mathbf{R}-\frac{2}{\operatorname{Re}_{\mathrm{ref}}} \sum_{j=1}^{3} \mathbf{R}^{T} \mathcal{B}_{1 j}^{s} \frac{\partial \mathbf{R}}{\partial x_{j}}\right]_{x=-1}^{1} d y d z \\
& -\frac{1}{\operatorname{Re}_{\mathrm{ref}}} \int_{\Omega}\left(\sum_{i=1}^{3} \sum_{j=i}^{3} \frac{\partial \mathbf{R}^{T}}{\partial x_{i}} \mathcal{B}_{i j}^{s} \frac{\partial \mathbf{R}}{\partial x_{j}}\right) d \Omega,
\end{aligned}
$$

where $\Omega=\Omega_{x} \times \Omega_{y} \times \Omega_{z}$. In deriving this expression, we use partial integration and assume the solution to be periodic or held at a constant value along the $y$ - and $z$-boundaries, i.e., contributions from these boundaries cancel. This is not a severe restriction, as this assumption is valid for a large variety of situations where open boundary conditions are applied.

It is evident that if we can prove

$$
\frac{1}{\operatorname{Re}_{\mathrm{ref}}} \int_{\Omega}\left(\sum_{i=1}^{3} \sum_{j=i}^{3} \frac{\partial \mathbf{R}^{T}}{\partial x_{i}} \mathcal{B}_{i j}^{s} \frac{\partial \mathbf{R}}{\partial x_{j}}\right) d \Omega \geq 0,
$$


then well-posedness may be ensured by properly constructing the boundary operator at the $x$-boundary.

Since the matrices, $\mathcal{B}_{i j}^{s}$, are all symmetric, (15) may be rewritten in a block-quadratic form as

$$
\frac{1}{2 \operatorname{Re}_{\mathrm{ref}}} \int_{\Omega} \tilde{\mathbf{R}}^{T} \mathcal{H}^{s} \tilde{\mathbf{R}} d \Omega \geq 0
$$

where we have introduced

$$
\tilde{\mathbf{R}}=\left[\frac{\partial \mathbf{R}}{\partial x}, \frac{\partial \mathbf{R}}{\partial y}, \frac{\partial \mathbf{R}}{\partial z}\right]^{T} \quad, \quad \mathcal{H}^{s}=\left[\begin{array}{ccc}
2 \mathcal{B}_{11}^{s} & \mathcal{B}_{12}^{s} & \mathcal{B}_{13}^{s} \\
\mathcal{B}_{12}^{s} & 2 \mathcal{B}_{22}^{s} & \mathcal{B}_{23}^{s} \\
\mathcal{B}_{13}^{s} & \mathcal{B}_{23}^{s} & 2 \mathcal{B}_{33}^{s}
\end{array}\right]
$$

We observe that $\mathcal{H}^{s}$ is a $15 \times 15$ symmetric block matrix, ensuring that the eigenvalue spectrum, $\rho\left(\mathcal{H}^{s}\right)$, is real. Hence, if $\mathcal{H}^{s}$ is positive semi-definite, (15) is obeyed. The eigenvalue spectrum, $\rho\left(\mathcal{H}^{s}\right)$, may be found to be

$$
\begin{aligned}
& \rho_{1}=\rho_{2}=\rho_{3}=0, \\
& \rho_{4}=2\left(\mu_{0}-\lambda_{0}\right), \\
& \rho_{5}=\rho_{6}=2\left(\lambda_{0}+3 \mu_{0}\right), \\
& \rho_{7}=\rho_{8}=3 \mu_{0}-\sqrt{\mu_{0}^{2}+2\left(\mu_{0}+\lambda_{0}\right)^{2}}, \\
& \rho_{9}=\rho_{10}=3 \mu_{0}+\sqrt{\mu_{0}^{2}+2\left(\mu_{0}+\lambda_{0}\right)^{2}}, \\
& \rho_{11}=7 \mu_{0}+4 \lambda_{0}-\sqrt{\mu_{0}^{2}+4\left(\mu_{0}+\lambda_{0}\right)\left(3 \mu_{0}+2 \lambda_{0}\right)} \\
& \rho_{12}=7 \mu_{0}+4 \lambda_{0}+\sqrt{\mu_{0}^{2}+4\left(\mu_{0}+\lambda_{0}\right)\left(3 \mu_{0}+2 \lambda_{0}\right)}, \\
& \rho_{13}=\rho_{14}=\rho_{15}=\left(\frac{2 c_{0}^{2}}{(\gamma-1)^{2}}+1\right) \frac{2(\gamma-1) k_{0}}{\operatorname{Pr}} .
\end{aligned}
$$

Here subscript " 0 " signifies the parameter values in the uniform state around which we have linearized. For most real fluids under nonextreme conditions, it is true that $\mu_{0}$ is positive, $\lambda_{0}$ is negative, and the following relationship is obeyed [20]:

$$
\frac{\gamma \mu_{0}}{\operatorname{Pr}} \geq \lambda_{0}+2 \mu_{0} \geq \mu_{0}
$$

A simple investigation of the eigenvalues reveals that $\mathcal{H}^{s}$ is positive semi-definite under these conditions. Thus, (15) is true provided

$$
\mu_{0} \geq 0, \quad \lambda_{0} \leq 0, \quad \lambda_{0}+\mu_{0} \geq 0, \quad \frac{\gamma k_{0}}{\operatorname{Pr}} \geq 0, \quad \gamma \geq 1 .
$$

These conditions are only natural as discussed in [21]. In fact, if they are not obeyed, NavierStokes equations violate the second law of thermodynamics.

We now obtain that well-posedness is ensured under the additional condition

$$
\forall(y, z) \in \Omega_{y} \times \Omega_{z}-\frac{1}{2}\left[\mathbf{R}^{T} \mathcal{A}_{1}^{s} \mathbf{R}-\frac{2}{\operatorname{Re}_{\mathrm{ref}}} \sum_{j=1}^{3} \mathbf{R}^{T} \mathcal{B}_{1 j}^{s} \frac{\partial \mathbf{R}}{\partial x_{j}}\right]_{x=-1}^{1} \leq 0,
$$


with the solution being bounded as

$$
\frac{1}{2} \frac{d}{d t}\|\mathcal{Q R}\|^{2} \leq-\frac{1}{\operatorname{Re}_{\mathrm{ref}}} \int_{\Omega}\left(\sum_{i=1}^{3} \sum_{j=i}^{3} \frac{\partial \mathbf{R}^{T}}{\partial x_{i}} \mathcal{B}_{i j}^{s} \frac{\partial \mathbf{R}}{\partial x_{j}}\right) d \Omega \leq 0,
$$

where $\mathcal{Q} \mathbf{R}=\mathcal{Q} \mathcal{S}^{-1} \mathbf{q}$.

As stated in Lemma 5.1, appropriate boundary conditions at the $x$-boundary have to obey

$$
-\frac{1}{2}\left[\mathbf{R}^{T} \mathcal{A}_{1}^{s} \mathbf{R}-\frac{2}{\operatorname{Re}_{\mathrm{ref}}} \sum_{j=1}^{3} \mathbf{R}^{T} \mathcal{B}_{1 j}^{s} \frac{\partial \mathbf{R}}{\partial x_{j}}\right]_{-1}^{1} \leq 0 .
$$

We now define

$$
\mathcal{Q}^{T} \mathcal{Q} \mathbf{G}=\sum_{j=1}^{3} \mathcal{B}_{1 j}^{s} \frac{\partial \mathbf{R}}{\partial x_{j}}
$$

where

$$
\begin{aligned}
& G_{1}=\frac{k_{0}(\gamma-1)}{2 \rho_{0} \operatorname{Pr}} \frac{\partial \zeta_{1}}{\partial x}+\frac{\lambda_{0}+2 \mu_{0}}{2 \rho_{0}} \frac{\partial \zeta_{2}}{\partial x}+\frac{\lambda_{0}+\mu_{0}}{\rho_{0}}\left(\frac{\partial R_{2}}{\partial y}+\frac{\partial R_{4}}{\partial z}\right), \\
& G_{2}=\frac{\mu_{0}}{\rho_{0}} \frac{\partial R_{2}}{\partial x}+\frac{\lambda_{0}+\mu_{0}}{2 \rho_{0}} \frac{\partial \zeta_{2}}{\partial y}, \\
& G_{3}=-\frac{k_{0}(\gamma-1)}{2 \rho_{0} c_{0} \operatorname{Pr}} \frac{\partial \zeta_{1}}{\partial x}, \\
& G_{4}=\frac{\mu_{0}}{\rho_{0}} \frac{\partial R_{4}}{\partial x}+\frac{\lambda_{0}+\mu_{0}}{2 \rho_{0}} \frac{\partial \zeta_{2}}{\partial z}, \\
& G_{5}=\frac{k_{0}(\gamma-1)}{2 \rho_{0} \operatorname{Pr}} \frac{\partial \zeta_{1}}{\partial x}-\frac{\lambda_{0}+2 \mu_{0}}{2 \rho_{0}} \frac{\partial \zeta_{2}}{\partial x}-\frac{\lambda_{0}+\mu_{0}}{\rho_{0}}\left(\frac{\partial R_{2}}{\partial y}+\frac{\partial R_{4}}{\partial z}\right),
\end{aligned}
$$

where we, for simplicity, have introduced

$$
\zeta_{1}=R_{1}+R_{5}-\frac{2 c_{0}}{\gamma-1} R_{3}, \zeta_{2}=R_{1}-R_{5}
$$

Some physical meaning can be given to the components of $\mathbf{G}$. The three components $G_{1}, G_{3}$, and $G_{5}$ account for effects caused by the normal heat flux and stress, whereas the remaining two, $G_{2}$ and $G_{4}$, are a consequence of tangential stress at the boundary.

This formulation allows for rewriting the constraint on the boundary contribution as

$$
-\frac{1}{2} \mathcal{Q}^{T}\left[\mathbf{R}^{T} \Lambda \mathbf{R}-\frac{2}{\operatorname{Re}_{\mathrm{ref}}} \mathbf{R}^{T} \mathbf{G}\right]_{-1}^{1} \mathcal{Q} \leq 0
$$

where $\Lambda$ is the diagonal eigenvalue matrix obtained from the similarity transform. Because $\mathcal{Q}^{T} \mathcal{Q}$ is positive definite, this inequality may be reformulated as

$$
-\frac{1}{2}\left[\sum_{i=1}^{5} \lambda_{i}^{-1}\left(\left(\left|\lambda_{i}\right| R_{i}-\varepsilon \frac{\left|\lambda_{i}\right|}{\lambda_{i}} G_{i}\right)^{2}-\left(\varepsilon G_{i}\right)^{2}\right)\right]_{-1}^{1} \leq 0,
$$

where $\lambda_{i}$ are the wave speeds by which the characteristic variables are advected, as given by the diagonal elements of $\Lambda$, and we have introduced $\varepsilon=\mathrm{Re}_{\mathrm{ref}}{ }^{-1}$. This formulation makes it 
straightforward to devise inflow-outflow boundary conditions, which are maximal dissipative and ensure well-posedness of the complete problem.

We note in particular that this formulation takes into account the off-diagonal terms of the stress-tensor, which are neglected in most previous work [8-10]. These terms may be of importance if the artificial boundary is introduced into a strongly vortical region of the flow, e.g., a wake flow behind a blunt body.

Inflow boundary conditions. At $x=-1,(18)$ becomes

$$
\frac{1}{2} \sum_{i=1}^{5} \lambda_{i}^{-1}\left(\left(\left|\lambda_{i}\right| R_{i}-\varepsilon \frac{\left|\lambda_{i}\right|}{\lambda_{i}} G_{i}\right)^{2}-\left(\varepsilon G_{i}\right)^{2}\right) \leq 0 .
$$

Subsonic inflow: $\lambda_{1}>0, \lambda_{2}>0, \lambda_{3}>0, \lambda_{4}>0, \lambda_{5}<0$.

$$
\begin{aligned}
\lambda_{1} R_{1}-\varepsilon G_{1} & =0, \\
\lambda_{2} R_{2}-\varepsilon G_{2} & =0, \\
\lambda_{3} R_{3}-\varepsilon G_{3} & =0, \\
\lambda_{4} R_{4}-\varepsilon G_{4} & =0, \\
\varepsilon G_{5} & =0 .
\end{aligned}
$$

Supersonic inflow: $\lambda_{1}>0, \lambda_{2}>0, \lambda_{3}>0, \lambda_{4}>0, \lambda_{5}>0$.

$$
\begin{aligned}
& \lambda_{1} R_{1}-\varepsilon G_{1}=0, \\
& \lambda_{2} R_{2}-\varepsilon G_{2}=0, \\
& \lambda_{3} R_{3}-\varepsilon G_{3}=0, \\
& \lambda_{4} R_{4}-\varepsilon G_{4}=0, \\
& \lambda_{5} R_{5}-\varepsilon G_{5}=0 .
\end{aligned}
$$

Outflow boundary conditions. At $x=1,(18)$ becomes

$$
\frac{1}{2} \sum_{i=1}^{5}-\lambda_{i}^{-1}\left(\left(\left|\lambda_{i}\right| R_{i}-\varepsilon \frac{\left|\lambda_{i}\right|}{\lambda_{i}} G_{i}\right)^{2}-\left(\varepsilon G_{i}\right)^{2}\right) \leq 0 .
$$

Subsonic outflow: $\lambda_{1}>0, \lambda_{2}>0, \lambda_{3}>0, \lambda_{4}>0, \quad \lambda_{5}<0$.

$$
\begin{aligned}
\varepsilon G_{2} & =0, \\
\varepsilon G_{3} & =0, \\
\varepsilon G_{4} & =0, \\
\left|\lambda_{5}\right| R_{5}+\varepsilon G_{5} & =0 .
\end{aligned}
$$

Supersonic outflow: $\lambda_{1}>0, \lambda_{2}>0, \lambda_{3}>0, \lambda_{4}>0, \lambda_{5}>0$.

$$
\begin{aligned}
& \varepsilon G_{1}=0, \quad \varepsilon G_{2}=0 \text {, } \\
& \varepsilon G_{2}=0, \quad \varepsilon G_{3}=0 \text {, } \\
& \varepsilon G_{3}=0 \text {, or } \varepsilon G_{4}=0 \text {, } \\
& \varepsilon G_{4}=0 . \quad \varepsilon G_{5}=0 \text {. }
\end{aligned}
$$

We note that for both types of outflow boundary conditions, it is only necessary to specify four conditions, since $\varepsilon G_{3}=0 \Rightarrow \varepsilon G_{1}=-\varepsilon G_{5}$. Due to the special structure of $\mathbf{G}$ we also observe that adding an extra condition on $\varepsilon G_{1}$ at the outflow does not place extra conditions on the solution, since such a condition is redundant. This observation will be used later. 
It was shown by Strikwerda [22] that the proper number of boundary conditions for an incomplete, parabolic system as the compressible Navier-Stokes equations is $\mathbf{5}$ in the inflow region and 4 in the outflow region. Our result clearly conforms with that.

We also note that in the limit of infinite Reynolds number, these boundary conditions converge uniformly toward the well-known characteristic boundary conditions for the compressible Euler equations [23]. This property is important in order to avoid weak boundary layers of the order $\exp (-x / \varepsilon)$ (see [8]).

5.2. The semi-discrete scheme. Following the line of thought that led to the asymptotically stable scheme for Burgers equation, we now propose a penalty method for enforcing open boundary conditions to a Legendre collocation approximation of the compressible Navier-Stokes equations

$$
\begin{aligned}
\frac{\partial \mathbf{q}}{\partial t}+\frac{\partial \mathbf{F}}{\partial x}+\frac{\partial \mathbf{G}}{\partial y}+\frac{\partial \mathbf{H}}{\partial z} & =\frac{1}{\operatorname{Re}_{\mathrm{ref}}}\left(\frac{\partial \mathbf{F}_{v}}{\partial x}+\frac{\partial \mathbf{G}_{v}}{\partial y}+\frac{\partial \mathbf{H}_{v}}{\partial z}\right) \\
& -\tau_{1} Q^{-}(x) \mathcal{S}\left(\mathcal{R}^{-}\left(\mathbf{R}-\mathcal{S}^{-1} \mathbf{g}_{1}(t)\right)-\frac{1}{\operatorname{Re}_{\mathrm{ref}}} \mathcal{G}^{-} \mathbf{G}\right) \\
& -\tau_{2} Q^{+}(x) \mathcal{S}\left(\mathcal{R}^{+}\left(\mathbf{R}-\mathcal{S}^{-1} \mathbf{g}_{2}(t)\right)+\frac{1}{\operatorname{Re}_{\mathrm{ref}}} \mathcal{G}^{+} \mathbf{G}\right) .
\end{aligned}
$$

Here $Q^{-}(x)$ and $Q^{+}(x)$ are given by (9) and $\mathcal{S}$ is the right eigenvector matrix as given in the Appendix. The boundary conditions for the state vector are given through the two vectors, $\mathbf{g}_{1}(t)$ and $\mathbf{g}_{2}(t)$, which we for convenience assume to represent a uniform state outside of the computational domain. The four matrices $\mathcal{R}^{-}, \mathcal{R}^{+}, \mathcal{G}^{-}$, and $\mathcal{G}^{+}$are chosen to construct the appropriate boundary operator as derived in the previous section. Hence, we have for the inflow region

$$
\mathcal{R}^{-}=\left[\begin{array}{ccccc}
\lambda_{1} & 0 & 0 & 0 & 0 \\
0 & \lambda_{2} & 0 & 0 & 0 \\
0 & 0 & \lambda_{3} & 0 & 0 \\
0 & 0 & 0 & \lambda_{4} & 0 \\
0 & 0 & 0 & 0 & \alpha \lambda_{5}
\end{array}\right], \quad \mathcal{G}^{-}=\left[\begin{array}{lllll}
1 & 0 & 0 & 0 & 0 \\
0 & 1 & 0 & 0 & 0 \\
0 & 0 & 1 & 0 & 0 \\
0 & 0 & 0 & 1 & 0 \\
0 & 0 & 0 & 0 & 1
\end{array}\right],
$$

where $\alpha=0$ for subsonic conditions and $\alpha=1$ for supersonic conditions. Likewise we define

$$
\mathcal{R}^{+}=\left[\begin{array}{ccccc}
0 & 0 & 0 & 0 & 0 \\
0 & 0 & 0 & 0 & 0 \\
0 & 0 & 0 & 0 & 0 \\
0 & 0 & 0 & 0 & 0 \\
0 & 0 & 0 & 0 & \beta\left|\lambda_{5}\right|
\end{array}\right], \quad \mathcal{G}^{+}=\left[\begin{array}{lllll}
0 & 0 & 0 & 0 & 0 \\
0 & 1 & 0 & 0 & 0 \\
0 & 0 & 1 & 0 & 0 \\
0 & 0 & 0 & 1 & 0 \\
0 & 0 & 0 & 0 & 1
\end{array}\right]
$$

where $\beta=1$ for subsonic conditions and $\beta=0$ for supersonic outflow conditions.

We have to choose $\tau_{1}$ and $\tau_{2}$ such that the semi-discrete scheme is asymptotically stable. The proper choice is stated in the following lemma.

LEMMA 5.2. Assume there exists a solution, $\mathbf{q}$, which is periodic or held at a constant value at the $y$-and z-boundary, and that the fluid properties of the uniform state, $\mathbf{q}_{0}$, are constrained by

$$
\mu_{0} \geq 0, \quad \lambda_{0} \leq 0, \quad \lambda_{0}+\mu_{0} \geq 0, \quad \frac{\gamma k_{0}}{\operatorname{Pr}} \geq 0, \quad \gamma \geq 1,
$$

and related as

$$
\frac{\gamma \mu_{0}}{\operatorname{Pr}} \geq \lambda_{0}+2 \mu_{0} \geq \mu_{0}
$$


The linearized, constant coefficient version of the scheme given by (23) is asymptotically stable at the inflow if

$$
\frac{1}{\omega \kappa}(1+\kappa+\sqrt{1+\kappa}) \geq \tau_{1} \geq \frac{1}{\omega \kappa}(1+\kappa-\sqrt{1+\kappa}) .
$$

Here

$$
\kappa=\frac{\varepsilon}{2 \omega} \frac{\gamma k_{0}}{\operatorname{Pr} \rho_{0} u_{0}} .
$$

This result is independent of whether the inflow is subsonic or supersonic.

For supersonic outflow

$$
\frac{1}{\omega}\left(1+\sqrt{\frac{1}{\kappa}}\right) \geq \tau_{2} \geq \frac{1}{\omega}\left(1-\sqrt{\frac{1}{\kappa}}\right) .
$$

For subsonic outflow

$$
\frac{1}{\omega \kappa}(1+\kappa+\sqrt{1+\kappa}) \geq \tau_{2} \geq \frac{1}{\omega \kappa}(1+\kappa-\sqrt{1+\kappa}) .
$$

The solution to (23) is bounded in the form

$$
\frac{1}{2} \frac{d}{d t}\|\mathcal{Q R}\|_{N}^{2} \leq-\frac{1}{\operatorname{Re}_{\text {ref }}} \sum_{k=1}^{N-1} \omega_{k} \int_{\Omega_{y}} \int_{\Omega_{z}}\left[\sum_{i=1}^{3} \sum_{j=i}^{3} \frac{\partial \mathbf{R}^{T}}{\partial x_{i}} \mathcal{B}_{i j}^{s} \frac{\partial \mathbf{R}}{\partial x_{j}}\right]_{x=x_{k}} d y d z \leq 0
$$

Proof. Write (23) in its symmetrized, linearized, constant coefficient version

$$
\begin{aligned}
\mathcal{Q}^{T} \mathcal{Q} \frac{\partial \mathbf{R}}{\partial t}+\sum_{i=1}^{3} \mathcal{A}_{i}^{s} \frac{\partial \mathbf{R}}{\partial x_{i}}= & \frac{1}{\operatorname{Re}_{\mathrm{ref}}} \sum_{i=1}^{3} \sum_{j=i}^{3} \mathcal{B}_{i j}^{s} \frac{\partial^{2} \mathbf{R}}{\partial x_{i} \partial x_{j}} \\
& -\tau_{1} Q^{-}(x)\left(\mathcal{Q}^{T} \mathcal{Q} \mathcal{R}^{-} \mathbf{R}-\frac{1}{\operatorname{Re}_{\mathrm{ref}}} \mathcal{Q}^{T} \mathcal{Q} \mathcal{G}^{-} \mathbf{G}\right) \\
& -\tau_{2} Q^{+}(x)\left(\mathcal{Q}^{T} \mathcal{Q} \mathcal{R}^{+} \mathbf{R}+\frac{1}{\operatorname{Re}_{\mathrm{ref}}} \mathcal{Q}^{T} \mathcal{Q} \mathcal{G}^{+} \mathbf{G}\right),
\end{aligned}
$$

where, without loss of generality, we have assumed homogeneous boundary conditions. We construct the energy integral, apply the Gauss-Lobatto quadrature rule, and use partial integration to obtain

$$
\begin{aligned}
\frac{1}{2} \frac{d}{d t}\|\mathcal{Q R}\|_{N}^{2} & =\int_{\Omega_{y}} \int_{\Omega_{z}}-\frac{1}{2}\left[\mathbf{R}^{T} \mathcal{A}_{1}^{s} \mathbf{R}-2 \varepsilon \sum_{j=1}^{3} \mathbf{R}^{T} \mathcal{B}_{1 j}^{s} \frac{\partial \mathbf{R}}{\partial x_{j}}\right]_{x=-1}^{1} d y d z \\
& -\varepsilon \int_{\Omega}\left(\sum_{i=1}^{3} \sum_{j=i}^{3} \frac{\partial \mathbf{R}^{T}}{\partial x_{i}} \mathcal{B}_{i j}^{s} \frac{\partial \mathbf{R}}{\partial x_{j}}\right) d \Omega \\
& -\tau_{1} \omega \int_{\Omega_{y}} \int_{\Omega_{z}}\left[\mathbf{R}^{T} \mathcal{Q}^{T} \mathcal{Q} \mathcal{R}^{-} \mathbf{R}-\varepsilon \mathbf{R}^{T} \mathcal{G}^{-} \sum_{j=1}^{3} \mathcal{B}_{1 j}^{s} \frac{\partial \mathbf{R}}{\partial x_{j}}\right]_{x=-1} d y d z \\
& -\tau_{2} \omega \int_{\Omega_{y}} \int_{\Omega_{z}}\left[\mathbf{R}^{T} \mathcal{Q}^{T} \mathcal{Q} \mathcal{R}^{+} \mathbf{R}+\varepsilon \mathbf{R}^{T} \mathcal{G}^{+} \sum_{j=1}^{3} \mathcal{B}_{1_{j}}^{s} \frac{\partial \mathbf{R}}{\partial x_{j}}\right]_{x=1} d y d z
\end{aligned}
$$


where we have used the assumption about periodicity or constant value at the $y$ - and $z$ boundary. Additionally, we have introduced $\varepsilon=\operatorname{Re}_{\text {ref }}{ }^{-1}$ and $\omega$, which is the Legendre weight at the endpoints and applied the definition

$$
\mathcal{Q}^{T} \mathcal{Q} \mathbf{G}=\sum_{j=1}^{3} \mathcal{B}_{1 j}^{s} \frac{\partial \mathbf{R}}{\partial x_{j}}
$$

Using the Gauss-Lobatto quadrature rule allows us to write

$$
\begin{aligned}
\int_{\Omega}\left(\sum_{i=1}^{3} \sum_{j=i}^{3} \frac{\partial \mathbf{R}^{T}}{\partial x_{i}} \mathcal{B}_{i j}^{s} \frac{\partial \mathbf{R}}{\partial x_{j}}\right) d \Omega= & \int_{\Omega_{y}} \int_{\Omega_{z}}\left(\sum_{k=1}^{N-1} \omega_{k}\left[\sum_{i=1}^{3} \sum_{j=i}^{3} \frac{\partial \mathbf{R}^{T}}{\partial x_{i}} \mathcal{B}_{i j}^{s} \frac{\partial \mathbf{R}}{\partial x_{j}}\right]_{x=x_{k}}\right. \\
& +\omega\left[\sum_{i=1}^{3} \sum_{j=i}^{3} \frac{\partial \mathbf{R}^{T}}{\partial x_{i}} \mathcal{B}_{i j}^{s} \frac{\partial \mathbf{R}}{\partial x_{j}}\right]_{x=-1} \\
& \left.+\omega\left[\sum_{i=1}^{3} \sum_{j=i}^{3} \frac{\partial \mathbf{R}^{T}}{\partial x_{i}} \mathcal{B}_{i j}^{s} \frac{\partial \mathbf{R}}{\partial x_{j}}\right]_{x=1}\right) d y d z \geq 0 .
\end{aligned}
$$

Here $x_{k}$ signifies the Legendre-Gauss-Lobatto collocation points. The inequality follows from the analysis done in the proof of Lemma 5.1 and is ensured provided the fluid properties are constrained by

$$
\mu_{0} \geq 0, \quad \lambda_{0} \leq 0, \quad \lambda_{0}+\mu_{0} \geq 0, \quad \frac{\gamma k_{0}}{\operatorname{Pr}} \geq 0, \quad \gamma \geq 1 .
$$

It was shown by Abarbanel and Gottlieb [20] that if a scheme is stable without the contributions from the off-diagonal stress-tensor terms, then it will remain so even if the these terms are included. This is a consequence of the general relation

$$
\frac{\gamma \mu_{0}}{\operatorname{Pr}} \geq \lambda_{0}+2 \mu_{0} \geq \mu_{0}
$$

which roughly gives the relation between the eigenvalues of the normal stress-tensor elements and the off-diagonal elements. Thus, it is sufficient to prove stability in the absence of the off-diagonal contributions.

The penalty parameters, $\tau_{1}$ and $\tau_{2}$, must be chosen such that the boundary term of the energy integral does not destroy the stability of the Cauchy problem. We treat the two boundary contributions separately.

Inflow condition. The contribution of the boundary term at the inflow $(x=-1)$ follows from combining (24) and (25) and neglecting the off-diagonal contributions to obtain

$$
\mathbf{R}^{T}\left(\frac{1}{2} \mathcal{A}_{1}^{s}-\tau_{1} \omega \mathcal{Q}^{T} \mathcal{Q} \mathcal{R}^{-}\right) \mathbf{R}-\varepsilon \mathbf{R}^{T}\left(\mathcal{I}-\tau_{1} \omega \mathcal{G}^{-}\right) \mathcal{B}_{11}^{s} \frac{\partial \mathbf{R}}{\partial x_{1}}-\varepsilon \omega \sum_{i=1}^{3} \frac{\partial \mathbf{R}^{T}}{\partial x_{i}} \mathcal{B}_{i i}^{s} \frac{\partial \mathbf{R}}{\partial x_{i}} \leq 0,
$$

where $\mathcal{I}$ is the identity matrix.

First we note that

$$
-\varepsilon \omega \frac{\partial \mathbf{R}^{T}}{\partial x_{2}} \mathcal{B}_{22}^{s} \frac{\partial \mathbf{R}}{\partial x_{2}}-\varepsilon \omega \frac{\partial \mathbf{R}^{T}}{\partial x_{3}} \mathcal{B}_{33}^{s} \frac{\partial \mathbf{R}}{\partial x_{3}} \leq 0
$$

since $\mathcal{B}_{22}^{s}$ and $\mathcal{B}_{33}^{s}$ are positive semi-definite with an eigenvalue spectrum given as

$$
\begin{array}{ll}
\rho_{1}\left(\mathcal{B}_{22}^{s}\right)=\rho_{1}\left(\mathcal{B}_{33}^{s}\right)=0, & \rho_{2}\left(\mathcal{B}_{22}^{s}\right)=\rho_{2}\left(\mathcal{B}_{33}^{s}\right)=\frac{\mu_{0}}{\rho_{0}}, \\
\rho_{3}\left(\mathcal{B}_{22}^{s}\right)=\rho_{3}\left(\mathcal{B}_{33}^{s}\right)=2 \frac{\mu_{0}}{\rho_{0}}, & \rho_{4}\left(\mathcal{B}_{22}^{s}\right)=\rho_{4}\left(\mathcal{B}_{33}^{s}\right)=2 \frac{\lambda_{0}+2 \mu_{0}}{\rho_{0}}, \\
\rho_{5}\left(\mathcal{B}_{22}^{s}\right)=\left(\frac{2 c_{0}^{2}}{(\gamma-1)^{2}}+1\right) \frac{(\gamma-1) k_{0}}{\rho_{0} \mathrm{Pr}}, & \rho_{5}\left(\mathcal{B}_{33}^{s}\right)=\left(\frac{2 c_{0}^{2}}{(\gamma-1)^{2}}+1\right) \frac{(\gamma-1) k_{0}}{\rho_{0} \mathrm{Pr}} .
\end{array}
$$


Since all matrices are symmetric, the remaining part of the constraint may be expressed in block-quadratic form as

$$
\tilde{\mathbf{R}}^{T} \mathcal{H}^{-} \tilde{\mathbf{R}} \leq 0
$$

where

$$
\tilde{\mathbf{R}}=\left[\mathbf{R}, \frac{\partial \mathbf{R}}{\partial x}\right]^{T}, \quad \mathcal{H}^{-}=\frac{1}{2}\left[\begin{array}{cc}
\mathcal{A}_{1}^{s}-2 \tau_{1} \omega \mathcal{Q}^{T} \mathcal{Q R}^{-} & -\varepsilon\left(1-\tau_{1} \omega\right) \mathcal{B}_{11}^{s} \\
-\varepsilon\left(1-\tau_{1} \omega\right) \mathcal{B}_{11}^{s} & -2 \varepsilon \omega \mathcal{B}_{11}^{s}
\end{array}\right]
$$

where we have used $\mathcal{G}^{-}=\mathcal{I}$. $\mathcal{H}^{-}$is a $10 \times 10$ symmetric block-matrix. Similar to the approach applied in $\S 3.2$, we have transformed the problem of stability into proving that $\mathcal{H}^{-}$, for a suitable value of $\tau_{1}$, is negative semi-definite. The eigenvalue-spectrum, $\rho\left(\mathcal{H}^{-}\right)$, can be found by doing an LU-decomposition. Since $\mathcal{H}^{-}$is symmetric, the eigenvalues appear as $\rho_{i}\left(\mathcal{H}^{-}\right)=U_{i i}$, i.e., the diagonal elements of the upper triangular matrix.

We will not give the general form of the eigenvalues here, since they are rather complicated. However, straightforward but very lengthy algebra shows that all eigenvalues are negative if $\tau_{1}$ is chosen such that

$$
\frac{1}{\omega \kappa}(1+\kappa+\sqrt{1+\kappa}) \geq \tau_{1} \geq \frac{1}{\omega \kappa}(1+\kappa-\sqrt{1+\kappa}),
$$

where

$$
\kappa=\frac{\varepsilon}{2 \omega} \frac{\gamma k_{0}}{\operatorname{Pr} \rho_{0} u_{0}} .
$$

This result is independent of whether the inflow is subsonic or supersonic.

Outflow condition. Neglecting the contribution from the off-diagonal terms yields a criteria for stability at the outflow $(x=1)$

$$
-\mathbf{R}^{T}\left(\frac{1}{2} \mathcal{A}_{1}^{s}+\tau_{2} \omega \mathcal{Q}^{T} \mathcal{Q} \mathcal{R}^{+}\right) \mathbf{R}+\varepsilon \mathbf{R}^{T}\left(\mathcal{I}-\tau_{2} \omega \mathcal{G}^{+}\right) \mathcal{B}_{11}^{s} \frac{\partial \mathbf{R}}{\partial x_{1}}-\varepsilon \omega \sum_{i=1}^{3} \frac{\partial \mathbf{R}^{T}}{\partial x_{i}} \mathcal{B}_{i i}^{s} \frac{\partial \mathbf{R}}{\partial x_{i}} \leq 0
$$

Similar to the approach followed in the previous part of the proof, we see that the contributions from $\mathcal{B}_{22}^{s}$ and $\mathcal{B}_{33}^{s}$ are always negative and independently ensure stability.

We now rewrite the remaining part of the condition at the outflow in block-quadratic form:

$$
\tilde{\mathbf{R}}^{T} \mathcal{H}^{+} \tilde{\mathbf{R}} \leq 0
$$

where

$$
\mathcal{H}^{+}=\frac{1}{2}\left[\begin{array}{cc}
-\mathcal{A}_{1}^{s}-2 \tau_{2} \omega \mathcal{Q}^{T} \mathcal{Q} \mathcal{R}^{+} & \varepsilon\left(1-\tau_{2} \omega\right) \mathcal{B}_{11}^{s} \\
\varepsilon\left(1-\tau_{1} \omega\right) \mathcal{B}_{11}^{s} & -2 \varepsilon \omega \mathcal{B}_{11}^{s}
\end{array}\right]
$$

To form $\mathcal{H}^{+}$we have assumed $\mathcal{G}^{+}=\mathcal{I}$. The additional boundary condition introduced by this replacement is redundant, as discussed in $\S 5.1$, and, hence, no extra restrictions are put on the system by this approach. The eigenvalue spectrum, $\rho\left(\mathcal{H}^{+}\right)$, may again be found through an LU-decomposition. We state here only the bounds on $\tau_{2}$ that ensure negative semi-definiteness of $\mathcal{H}^{+}$for supersonic outflow

$$
\frac{1}{\omega}\left(1+\sqrt{\frac{1}{\kappa}}\right) \geq \tau_{2} \geq \frac{1}{\omega}\left(1-\sqrt{\frac{1}{\kappa}}\right) .
$$


For subsonic outflow the bounds become

$$
\frac{1}{\omega \kappa}(1+\kappa+\sqrt{1+\kappa}) \geq \tau_{2} \geq \frac{1}{\omega \kappa}(1+\kappa-\sqrt{1+\kappa}) .
$$

Combining (24) and (25), we obtain a bound for the growth of the solution

$$
\frac{1}{2} \frac{d}{d t}\|\mathcal{Q R}\|_{N}^{2} \leq-\frac{1}{\operatorname{Re}_{\mathrm{ref}}} \sum_{k=1}^{N-1} \omega_{k} \int_{\Omega_{y}} \int_{\Omega_{z}}\left[\sum_{i=1}^{3} \sum_{j=i}^{3} \frac{\partial \mathbf{R}^{T}}{\partial x_{i}} \mathcal{B}_{i j}^{s} \frac{\partial \mathbf{R}}{\partial x_{j}}\right]_{x=x_{k}} d y d z \leq 0 .
$$

We wish to emphasize that the bounds on $\tau_{1}$ and $\tau_{2}$ given in Lemma 5.2 remain valid in the limit when the Reynolds number approaches infinity. This is easily realized by expanding the bounds for $\varepsilon \ll 1$ to obtain

$$
\infty>\tau_{1} \geq \frac{1}{2 \omega}+\varepsilon \frac{1}{8 \omega} \kappa
$$

in the inflow region and

$$
\infty>\tau_{2}>-\infty, \infty>\tau_{2} \geq \frac{1}{2 \omega}+\varepsilon \frac{1}{8 \omega} \kappa
$$

for supersonic and subsonic outflow, respectively. The linearized, constant coefficient version of the Euler equations may be transformed into 5 independent hyperbolic equations for which we should expect the bounds on the penalty parameters to be given by the results in $\S 3.1 .1$. We observe that the bounds given above converge uniformly to the expected values in the limit of vanishing viscosity and, thus, the scheme remains stable. The observation that no bounds are necessary on $\tau_{2}$ for supersonic outflow simply reflects the fact that no boundary conditions are required for the Euler equations at such a boundary.

5.3. Numerical tests. The proof given in the previous section is only strictly valid for the linearized, constant coefficient version of Navier-Stokes equations. To validate the results and show that it carries over to full nonlinear Navier-Stokes equations, we have implemented the scheme in an existing spectral code (see [24] for details), originally developed for studying two-dimensional compressible flow around an infinitely long circular cylinder.

For the spatial approximation scheme we used a standard Chebyshev-Fourier collocation scheme in polar coordinates, $(r, \theta)$, with a third-order Runge-Kutta method for time-stepping.

The new scheme is simple to implement in existing codes, because we only need to apply a correction of the flux of the state vector at the boundary. Following the scheme, given by (23), we need to derive the two vectors $\mathbf{R}$ and $\mathbf{G}$. The characteristic variables are given as

$$
\begin{aligned}
& R_{1}=\left(m_{r}-\rho u_{r}\right)+\frac{p}{c_{0}}, \\
& R_{2}=m_{\theta}-\rho u_{\theta}, \\
& R_{3}=\rho-\frac{p}{c_{0}^{2}}, \\
& R_{4}=-\left(m_{r}-\rho u_{r}\right)+\frac{p}{c_{0}},
\end{aligned}
$$

where $c_{0}$ is the uniform state sound speed.

We have for convenience introduced

$$
u_{r}=u_{0} \hat{k}_{1}+v_{0} \hat{k}_{2}, \quad u_{\theta}=u_{0} \hat{k}_{2}-v_{0} \hat{k}_{1}
$$


which are the radial and azimuthal velocity components, respectively, of the uniform state, and

$$
m_{r}=m_{u} \hat{k}_{1}+m_{v} \hat{k}_{2}, \quad m_{\theta}=m_{u} \hat{k}_{2}-m_{v} \hat{k}_{1},
$$

which are the radial and azimuthal components of the momentum of the flow field. Here $\mathbf{k}=\left(\hat{k}_{1}, \hat{k}_{2}\right)$ signifies an outward pointing normal-vector at the boundary. The linearized pressure, $p$, is given as

$$
p=(\gamma-1)\left[E+\frac{1}{2} \rho\left(u_{0}^{2}+v_{0}^{2}\right)-u_{0} m_{u}-v_{0} m_{v}\right] .
$$

The eigenvalues corresponding to the characteristic functions and determining the direction and propagation velocity of the characteristic waves are

$$
\lambda_{1}=u_{r}+c_{0}, \lambda_{2}=\lambda_{3}=u_{r}, \lambda_{4}=u_{r}-c_{0} .
$$

Following the approach outlined in the previous section, we have likewise derived the viscous correction vector, $\mathbf{G}$, at the outer boundary as

$$
\begin{aligned}
& G_{1}=\frac{1}{\rho_{0} r}\left[\frac{(\gamma-1) k_{0}}{2 \operatorname{Pr}} \frac{\partial\left(r \zeta_{1}\right)}{\partial r}+\frac{2}{3} \mu_{0} \frac{\partial\left(r \zeta_{2}\right)}{\partial r}-\frac{1}{3} \mu_{0} \frac{\partial R_{2}}{\partial \theta}\right], \\
& G_{2}=\frac{\mu_{0}}{\rho_{0} r}\left[\frac{\partial\left(r R_{2}\right)}{\partial r}-\frac{1}{6} \frac{\partial \zeta_{2}}{\partial \theta}\right], \\
& G_{3}=-\frac{(\gamma-1) k_{0}}{\operatorname{Pr}} \frac{1}{2 c_{0} \rho_{0}} \frac{1}{r} \frac{\partial\left(r \zeta_{1}\right)}{\partial r}, \\
& G_{4}=\frac{1}{\rho_{0} r}\left[\frac{(\gamma-1) k_{0}}{2 \operatorname{Pr}} \frac{\partial\left(r \zeta_{1}\right)}{\partial r}-\frac{2}{3} \mu_{0} \frac{\partial\left(r \zeta_{2}\right)}{\partial r}+\frac{1}{3} \mu_{0} \frac{\partial R_{2}}{\partial \theta}\right],
\end{aligned}
$$

where again we have defined

$$
\zeta_{1}=R_{1}+R_{4}-\frac{2 c_{0}}{\gamma-1} R_{2}, \quad \zeta_{2}=R_{1}-R_{4},
$$

and applied Stokes hypothesis to obtain $\lambda=-\frac{2}{3} \mu$. We note that only two extra calculations of derivatives, $(\partial \rho / \partial r, \partial \rho / \partial \theta)$, are needed in order to form the two vectors, since the radial and azimuthal derivatives at the boundary of the remaining variables are calculated during evaluation of the interior dynamics when employing a global scheme. Thus, compared with evaluation of the flux, the computational requirement for enforcing the boundary conditions through this new method is negligible.

The boundary conditions are enforced at each intermediate time-step of the Runge-Kutta method. Simulations were done with a Reynolds number of 100, a Mach number of 0.4, a diameter $(L)$ of the cylinder of $6.10 \mathrm{~mm}$, and a reference temperature of $300^{\circ} \mathrm{K}$. These parameters ensure that the flow field remains subsonic. The resolution was 96 Fourier modes, 72 Chebyshev modes and the radius $\left(L_{D}\right)$ of the computational domain was 20 cylinder diameters.

As penalty parameters we used

$$
\tau_{1}=\tau_{2}=\frac{N^{2}}{4 \kappa} \frac{2}{L_{D}}(1+\kappa-\sqrt{1+\kappa}),
$$

where $N$ is the number of Chebyshev modes, $2 / L_{D}$ is a result of the radial mapping of $L_{D}$ into $[-1,1]$, and

$$
\kappa=\frac{\varepsilon N^{2}}{2} \frac{\gamma k_{0}}{\operatorname{Pr} \rho_{0}\left|u_{r}\right|},
$$



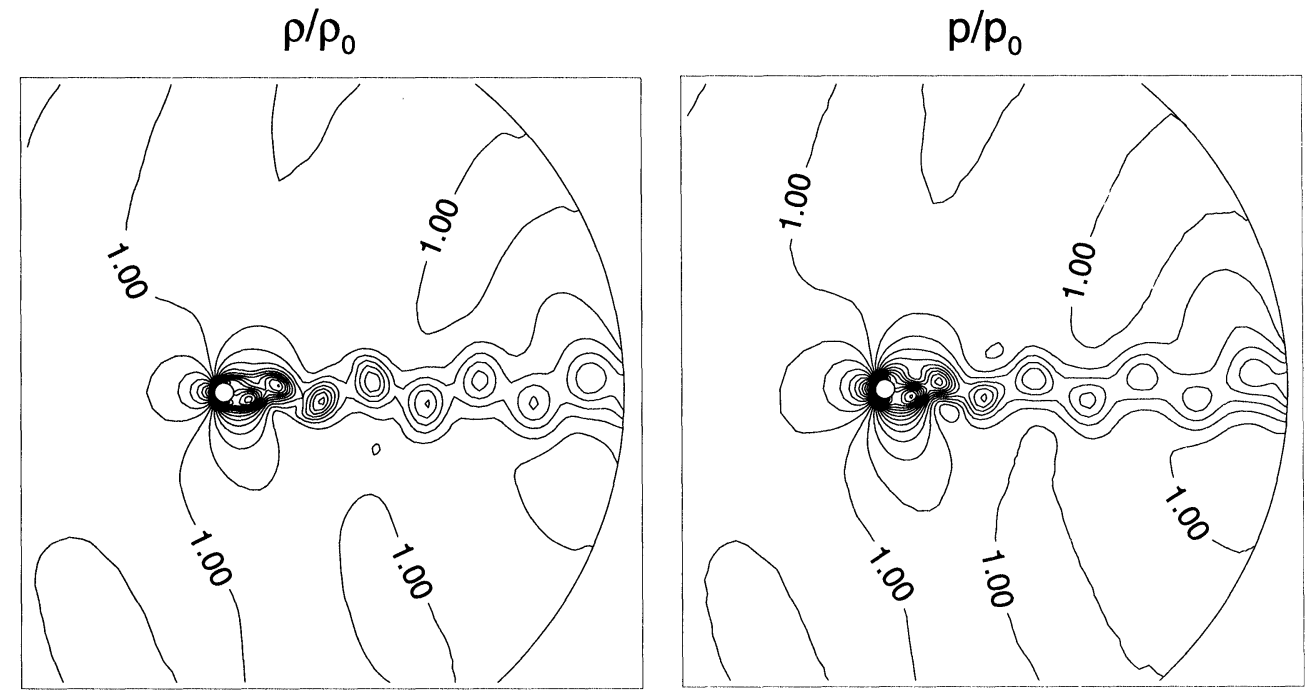

FIG. 4. Contour plots of the normalized density, $\rho / \rho_{0}$, and the normalized pressure, $p / p_{0}$, at the nondimensional time $T=143.5$ for a flow at $\mathrm{Re}=100, \mathrm{M}=0.4, D=6.10 \mathrm{~mm}$, and $T_{0}=300^{\circ} \mathrm{K}$.

This choice appears natural from the results stated in Lemma 5.2 and the experience gained in $\S 4$, indicating that for dissipative problems we may reduce the penalty parameter by a factor of 4 to obtain the optimal value of $\tau$. With this choice of penalty parameters we were able to perform the simulations without any reduction in time-step as compared with the exact method of enforcing the boundary conditions. It should be mentioned that in the original code only characteristic boundary conditions for the Euler equations were enforced. Comparing with results discussed in $\S 4$, we observe that for third-order Runge-Kutta methods we should expect the two methods to impose almost equivalent time-step restrictions. This is confirmed by the simulations and shows that the results from the simple linear analysis carries over to the full nonlinear Navier-Stokes equations in this case.

In Fig. 4 we show contour plots of the normalized density and the pressure at $T=143.5$, corresponding to approximately 23 shedding cycles. The von Karman vortex street is clearly demonstrated, and we observe that the boundary conditions at the outflow boundary affect the flow only slightly. The Strouhal number for the shredding frequency is found to be $S t=0.163$, which is in full accordance with experimental findings [25] and we observe no spurious frequencies or reflections from the artificial boundary back into the flow field (see [24] for a further discussion of this).

6. Concluding remarks. The purpose of the present paper has been twofold. The first goal has been to develop boundary conditions for wave-dominated problems, leading to wellposed total problems. It was argued that for smooth solutions and the class of operators we have considered here, it is sufficient to consider the problem of well-posedness for the locally linearized, constant coefficient version of the nonlinear initial-boundary value problem. Using this allowed the derivation of proper boundary conditions to the Burgers equation and to the three-dimensional, compressible Navier-Stokes equations, and these boundary conditions were shown to ensure well-posedness of the total problem. It should be stressed that the boundary conditions derived for the Navier-Stokes equations take into account all elements of the stress-tensor, and only very light assumptions were made to derive these. Additionally, they remain valid even in the limit of vanishing viscosity. 
Having derived appropriate boundary conditions naturally leads to the question of how to enforce these in a discrete approximation of the problem. This has been the second, and main, contribution of the paper. Results [7] on the connection between stability of discrete and semi-discrete approximations suggest that it is sufficient to consider asymptotic stability for the semi-discrete approximation. We have only considered Legendre collocation methods here. This choice is dictated merely by a wish to obtain analytical results and we have indicated, by numerical tests, that all results carry over to Chebyshev collocation methods. The stability proofs for the semi-discrete approximations to the linearized, constant coefficient versions of the Burgers equation and the compressible Navier-Stokes equations are all completed by using the classical energy method. We emphasized that the proposed schemes remain stable even in the limit where the problems become purely hyperbolic.

The proposed penalty method changes the eigenvalue spectra of the discrete approximations of the operators considerably. In order to understand this, we performed a detailed investigation of the effect of the penalty method on the eigenvalue spectra of linear operators. It has been shown that the value of the penalty parameter, which is obtained from the theoretical analysis, often implies that the maximum allowable time-step compares unfavorably with that allowed for more traditional methods. However, we discussed in detail how to remedy this and showed that choosing the penalty parameter properly may in some cases allow for increasing the maximum time-step by as much as $50 \%$. Although we are not aware of a systematic way of determining the optimal value of the penalty parameter, we do not see that as a significant disadvantage. Our experience tells that once the theoretical values of the penalty parameters are obtained, only a few tests are needed to obtain the optimal value. Additionally, this has to be done only once, and since only a few hundred time-steps are required to test whether the scheme is stable, we consider this an insignificant problem.

Most of the theoretical results, obtained for linearized, constant coefficient versions of the equations, are confirmed by numerical simulations of the full nonlinear equations. It is stressed that the proposed penalty method is very easy to implement in existing codes, which is an attractive feature.

Although all results and numerical simulations in this paper are obtained using spectral collocation methods, the main conclusions carry over to finite difference/finite element methods. The derivation of the proper boundary operators, for either the Burgers equation or for the compressible, Navier-Stokes equations, is obviously unaffected by the choice of the spatial approximation method. The proposed penalty method for enforcing the boundary conditions may be applied in exactly the same manner as discussed here when using alternative spatial discretization methods. The only difference is the value of the penalty parameter, which will depend strongly on the order of the method. Thus, applying another method requires one to derive this penalty parameter. This may be done by an approach equivalent to the one utilized here.

In a future paper [26], we will extend the penalty method developed here to include multidomain solutions of the compressible Navier-Stokes equations in general curvilinear coordinates.

Appendix: Symmetric matrices for the Navier-Stokes equations. Consider the linearized, constant coefficient compressible Navier-Stokes equations in conservation form given as

$$
\frac{\partial \mathbf{q}}{\partial t}+\sum_{i=1}^{3} \mathcal{A}_{i} \frac{\partial \mathbf{q}}{\partial x_{i}}=\frac{1}{\operatorname{Re}_{\mathrm{ref}}} \sum_{i=1}^{3} \sum_{j=i}^{3} \mathcal{B}_{i j} \frac{\partial^{2} \mathbf{q}}{\partial x_{i} \partial x_{j}}
$$


The matrix, $\mathcal{A}_{1}$, diagonalizes under the similarity transform, $\Lambda=\mathcal{S}^{-1} \mathcal{A}_{1} \mathcal{S}$, where the right eigenvector matrix, $\mathcal{S}$, and the left eigenvector matrix, $\mathcal{S}^{-1}$, are given as

$$
\begin{gathered}
\mathcal{S}=\left[\begin{array}{ccccc}
\alpha & 0 & 1 & 0 & \alpha \\
\alpha(u+c) & 0 & u & 0 & \alpha(u-c) \\
\alpha v & 1 & v & 0 & \alpha v \\
\alpha w & 0 & w & 1 & \alpha w \\
\alpha(H+c u) & v & \frac{1}{2} c^{2} M^{2} & w & \alpha(H-c u)
\end{array}\right], \\
\mathcal{S}^{-1}=\left[\begin{array}{ccccc}
\beta\left(\frac{1}{2}(\gamma-1) c^{2} M^{2}-c u\right) & -\beta((\gamma-1) u-c) & -\beta(\gamma-1) v & -\beta(\gamma-1) w & \beta(\gamma-1) \\
-v & 0 & 1 & 0 & 0 \\
1-\frac{1}{2}(\gamma-1) M^{2} & \frac{\gamma-1}{c^{2}} u & \frac{\gamma-1}{c^{2}} v & \frac{\gamma-1}{c^{2}} w & -\frac{\gamma-1}{c^{2}} \\
-w & 0 & 0 & 1 & 0 \\
\beta\left(\frac{1}{2}(\gamma-1) c^{2} M^{2}+c u\right) & -\beta((\gamma-1) u+c) & -\beta(\gamma-1) v & -\beta(\gamma-1) w & \beta(\gamma-1)
\end{array}\right] .
\end{gathered}
$$

Here

$$
\alpha=\frac{1}{2 c}, \quad \beta=\frac{1}{c} \text {. }
$$

Introducing this transformation into the Navier-Stokes equations yields

$$
\mathcal{Q}^{T} \mathcal{Q} \frac{\partial \mathbf{R}}{\partial t}+\sum_{i=1}^{3} \mathcal{A}_{i}^{s} \frac{\partial \mathbf{R}}{\partial x_{i}}=\frac{1}{\operatorname{Re}_{\mathrm{ref}}} \sum_{i=1}^{3} \sum_{j=i}^{3} \mathcal{B}_{i j}^{s} \frac{\partial^{2} \mathbf{R}}{\partial x_{i} \partial x_{j}}
$$

where $\mathbf{R}$ are the characteristic variables and $\mathcal{Q}^{T} \mathcal{Q}$ is a positive definite, symmetrizing diagonal matrix.

The symmetrized matrices

$$
\mathcal{A}_{i}^{s}=\mathcal{Q}^{T} \mathcal{Q} \mathcal{S}^{-1} \mathcal{A}_{i} \mathcal{S}, \quad \mathcal{B}_{i j}^{s}=\mathcal{Q}^{T} \mathcal{Q} \mathcal{S}^{-1} \mathcal{B}_{i j} \mathcal{S}
$$

are given as

$$
\begin{gathered}
\mathcal{A}_{1}^{s}=\left[\begin{array}{ccccc}
u+c & 0 & 0 & 0 & 0 \\
0 & 2 u & 0 & 0 & 0 \\
0 & 0 & \frac{2 c^{2}}{\gamma-1} u & 0 & 0 \\
0 & 0 & 0 & 2 u & 0 \\
0 & 0 & 0 & 0 & u-c
\end{array}\right], \mathcal{A}_{2}^{s}=\left[\begin{array}{ccccc}
v & c & 0 & 0 & 0 \\
c & 2 v & 0 & 0 & c \\
0 & 0 & \frac{2 c^{2}}{\gamma-1} & 0 & 0 \\
0 & 0 & 0 & 2 v & 0 \\
0 & c & 0 & 0 & v
\end{array}\right], \\
\mathcal{A}_{3}^{s}=\left[\begin{array}{ccccc}
w & 0 & 0 & c & 0 \\
0 & 2 w & 0 & 0 & 0 \\
0 & 0 & \frac{2 c^{2}}{\gamma-1} w & 0 & 0 \\
c & 0 & 0 & 2 w & c \\
0 & 0 & 0 & c & w
\end{array}\right], \\
\mathcal{B}_{11}^{s}=\frac{1}{2 \rho}\left[\begin{array}{ccccc}
(\lambda+2 \mu)+\theta & 0 & -\frac{2 c}{\gamma-1} \theta & 0 & -(\lambda+2 \mu)+\theta \\
0 & 4 \mu & 0 & 0 & 0 \\
-\frac{2 c}{\gamma-1} \theta & 0 & \frac{4 c^{2}}{(\gamma-1)^{2}} \theta & 0 & -\frac{2 c}{\gamma-1} \theta \\
0 & 0 & 0 & 4 \mu & 0 \\
-(\lambda+2 \mu)+\theta & 0 & -\frac{2 c}{\gamma-1} \theta & 0 & (\lambda+2 \mu)+\theta
\end{array}\right]
\end{gathered}
$$




$$
\begin{gathered}
\mathcal{B}_{22}^{s}=\frac{1}{2 \rho}\left[\begin{array}{ccccc}
\mu+\theta & 0 & -\frac{2 c}{\gamma-1} \theta & 0 & -\mu+\theta \\
0 & 4(\lambda+2 \mu) & 0 & 0 & 0 \\
-\frac{2 c}{\gamma-1} \theta & 0 & \frac{4 c^{2}}{(\gamma-1)^{2}} \theta & 0 & -\frac{2 c}{\gamma-1} \theta \\
0 & 0 & 0 & 4 \mu & 0 \\
-\mu+\theta & 0 & -\frac{2 c}{\gamma-1} \theta & 0 & \mu+\theta
\end{array}\right], \\
\mathcal{B}_{33}^{s}=\frac{1}{2 \rho}\left[\begin{array}{ccccc}
\mu+\theta & 0 & -\frac{2 c}{\gamma-1} \theta & 0 & -\mu+\theta \\
0 & 4 \mu & 0 & 0 & 0 \\
-\frac{2 c}{\gamma-1} \theta & 0 & \frac{4 c^{2}}{(\gamma-1)^{2}} \theta & 0 & -\frac{2 c}{\gamma-1} \theta \\
0 & 0 & 0 & 4(\lambda+2 \mu) & 0 \\
-\mu+\theta & 0 & -\frac{2 c}{\gamma-1} \theta & 0 & \mu+\theta
\end{array}\right] .
\end{gathered}
$$

We have for convenience introduced

$$
\begin{gathered}
\theta=\frac{\gamma-1}{\gamma} \frac{\gamma k}{\operatorname{Pr}}, \\
\mathcal{B}_{12}^{s}=\frac{\lambda+\mu}{\rho}\left[\begin{array}{rrrrr}
0 & 1 & 0 & 0 & 0 \\
1 & 0 & 0 & 0 & -1 \\
0 & 0 & 0 & 0 & 0 \\
0 & 0 & 0 & 0 & 0 \\
0 & -1 & 0 & 0 & 0
\end{array}\right], \mathcal{B}_{13}^{s}=\frac{\lambda+\mu}{\rho}\left[\begin{array}{rrrrrr}
0 & 0 & 0 & 1 & 0 \\
0 & 0 & 0 & 0 & 0 \\
0 & 0 & 0 & 0 & 0 \\
1 & 0 & 0 & 0 & -1 \\
0 & 0 & 0 & -1 & 0
\end{array}\right], \\
\mathcal{B}_{23}^{s}=\frac{\lambda+\mu}{\rho}\left[\begin{array}{lllll}
0 & 0 & 0 & 0 & 0 \\
0 & 0 & 0 & 2 & 0 \\
0 & 0 & 0 & 0 & 0 \\
0 & 2 & 0 & 0 & 0 \\
0 & 0 & 0 & 0 & 0
\end{array}\right] .
\end{gathered}
$$

Acknowledgment. The authors would like to thank Dr. W. S. Don and Dr. L. Dettori from Brown University for many useful discussions.

\section{REFERENCES}

[1] H. O. KReISS AND J. LoRenZ, Initial-Boundary Value Problems and the Navier-Stokes Equations, Series in Pure and Applied Mathematics, Academic Press, San Diego, CA, 1989.

[2] A. HARTEN, On the symmetric form of systems of conservation laws with entropy, J. Comput. Phys., 49 (1983), pp. 151-164.

[3] D. FUNARO AND D. GoTtlieB, A new method of imposing boundary conditions in pseudospectral approximations of hyperbolic equations, Math. Comp., 51 (1988), pp. 599-613.

[4] - Convergence results for pseudospectral approximations of hyperbolic systems by a penalty-type boundary treatment, Math. Comp., 57 (1991), pp. 585-596.

[5] M. CARPENTER, D. GoTTLIEB, AND S. ABARBANEL, Time-stable boundary conditions for finite-difference schemes solving hyperbolic systems: Methodology and applications to high-order compact schemes, ICASE Report no. 93-9, 1993.

[6] W. S. DON AND D. GoTTLIEB, The Chebyshev-Legendre method: Implementing Legendre methods on Chebyshev points, SIAM J. Numer. Anal., 31 (1994), pp. 1519-1534.

[7] H. O. KREISS AND L. WU, On the stability definition of difference approximations for the initial boundary value problem, Appl. Numer. Math., 12 (1993), pp. 213-227.

[8] B. GuSTAFSSON AND A. SUNDSTRÖM, Incompletely parabolic problems in fluid dynamics, SIAM J. Appl. Math., 35 (1978), pp. 343-357.

[9] J. Oliger AND A. SUNDSTRÖM, Theoretical and practical aspects of some initial boundary value problems in fluid dynamics, SIAM J. Appl. Math., 35 (1978), pp. 419-446.

[10] J. Nordström, Artificial Boundary Conditions for the Navier-Stokes Equations, Ph.D. Thesis, Uppsala University, Sweden, 1993. 
[11] J. NORDSTRÖM, The use of characteristic boundary conditions for the Navier-Stokes equations, Comput. Fluids, 24 (1995), pp. 609-623.

[12] P. DuTT, Stable boundary conditions and difference schemes for Navier-Stokes equations, SIAM J. Numer. Anal., 25 (1988), pp. 245-267.

[13] L. HALPERN, Artificial boundary conditions for incompletely parabolic perturbations of hyperbolic systems, SIAM J. Math. Anal., 22 (1991), pp. 1256-1283.

[14] P. J. DaVIS AND P. RabinowitZ, Methods of Numerical Integration. 2nd ed., Academic Press, San Diego, 1984.

[15] D. GotTlieb, M. Y. Hussaini, AND S. A. OrsZAG, Introduction: Theory and applications of spectral methods, in Spectral Methods for Partial Differential Equations, R. Voigt, D. Gottlieb, and M.Y. Hussaini, eds., SIAM, Philadelphia, 1984, pp. 1-54.

[16] D. Funaro, Polynomial Approximation of Differential Equations, Springer-Verlag, New York, 1992.

[17] D. GotTlieb AND L. Lustman, The spectrum of the Chebyshev collocation operator for the heat equation, SIAM J. Numer. Anal., 20 (1983), pp. 909-921.

[18] C. Canuto, M. Y. Hussaini, A. QuarTeroni, And T. A. Zang, Spectral Methods in Fluid Dynamics, Springer Series in Computational Physics, Springer-Verlag, New York, 1988.

[19] D. GotTlieb, M. GUNZBURGER, AND E. TuRKEL, On numerical boundary treatment of hyperbolic systems for finite difference and finite element methods, SIAM J. Numer. Anal., 19 (1982), pp. 671-682.

[20] S. ABARBANEL AND D. GotTlieb, Optimal time splitting for two-and three-dimensional Navier-Stokes equations with mixed derivatives, J. Comput. Phys., 41 (1981), pp. 1-33.

[21] T. J. R. Hughes, L. P. FranCA, AND M. MALlEt, A new finite element formulation for computational fluid dynamics: I. Symmetric forms of the compressible Euler and Navier-Stokes equations and the second law of thermodynamics, Comput. Methods Appl. Mech. Engrg., 54 (1986), pp. 223-234.

[22] J. C. STRIKWERDA, Initial boundary value problems for incompletely parabolic systems, Comm. Pure Appl. Math., 30 (1977), pp. 797-822.

[23] K. W. ThOMPSON, Time-dependent boundary conditions for hyperbolic systems, J. Comput. Phys., 68 (1987), pp. 1-29.

[24] W. S. DON AND D. GoTTLIEB, Spectral simulation of an unsteady compressible flow past a circular cylinder, Comput. Methods Appl. Mech. Engrg., 80 (1990), pp. 39-58.

[25] C. H. K. Williamson, Oblique and parallel modes of vortex shedding in the wake of a circular cylinder at low Reynolds numbers, J. Fluid Mech., 206 (1989), pp. 579-627.

[26] J. S. HESTHAVEN, A stable penalty method for the compressible Navier-Stokes equations. II. One dimensional domain decomposition schemes, SIAM J. Sci. Comput., 18 (1997), to appear. 